DOI 10.4171/JEMS/381

Alexander Mielke $\cdot$ Ulisse Stefanelli

\title{
Linearized plasticity is the evolutionary $\Gamma$-limit of finite plasticity
}

Received June 14, 2011 and in revised form September 22, 2011

\begin{abstract}
We provide a rigorous justification of the classical linearization approach in plasticity. By taking the small-deformations limit, we prove via $\Gamma$-convergence for rate-independent processes that energetic solutions of the quasi-static finite-strain elastoplasticity system converge to the unique strong solution of linearized elastoplasticity.
\end{abstract}

Keywords. Finite-strain elastoplasticity, linearized elastoplasticity, $\Gamma$-convergence, rate-independent processes

\section{Introduction}

This paper is devoted to the rigorous justification of the classical linearization approach in finite-strain elastoplasticity. When restricting to the small-deformation realm it is indeed customary to leave the nonlinear finite-strain framework and resort to linearized theories instead. This reduction is usually motivated by means of heuristic Taylor expansion arguments. Here, we aim at complement these formal motivations by providing a rigorous linearization proof by means of an evolutionary $\Gamma$-convergence analysis of rateindependent processes. In particular, we address the general time-dependent case, which e.g. allows for cyclic loading.

In the stationary framework, the pioneering contribution in this context goes back to Dal Maso, Negri, \& Percivale [DNP02] who devised a proof of convergence of finitestrain elasticity to linearized elasticity. Later, the argument was refined by Agostiniani, Dal Maso, \& DeSimone [ADD12] and extended to multi-well energies by Schmidt [Sch08] and to residually stressed materials by Paroni \& Tomassetti [PT09, PT11]. The reader is also referred to [GN11, MN11, Neu10] for some related results on homogenization, to [AD11] for an application to the study of nematic elastomers, to [BSV07,

A. Mielke: Weierstraß-Institut für Angewandte Analysis und Stochastik, Mohrenstraße 39,

D-10117 Berlin and Institut für Mathematik, Humboldt-Universität zu Berlin,

Rudower Chaussee 25, D-12489 Berlin, Germany; e-mail: mielke@wias-berlin.de,

URL: http://www.wias-berlin.de/people/mielke/

U. Stefanelli: Istituto di Matematica Applicata e Tecnologie Informatiche E. Magenes - CNR,

v. Ferrata 1, I-27100 Pavia, Italy and Weierstraß-Institut für Angewandte Analysis und Stochastik, Mohrenstraße 39, D-10117 Berlin, Germany; e-mail: ulisse.stefanelli@imati.cnr.it,

URL: http://www.imati.cnr.it/ulisse/

Mathematics Subject Classification (2010): 74C15, 49J45 
Sch09] in the context of convergence of atomistic models, and to [SZ11] in relation with dislocation theory.

To our knowledge, this is the first result in the evolutionary case. Compared to the stationary case of [DNP02], the evolution situation is quite more involved. Indeed, the argument in [DNP02] relies on the $\Gamma$-convergence of the small-deformation energy functional to its linearization limit. Here, we are instead forced to cope with the occurrence of dissipative plastic evolution by means of a delicate recovery sequence construction relating energy and dissipation. We emphasize that finite-strain elastoplasticity is based on a multiplicative decomposition of strain tensors. Moreover, the plastic tensor is to be considered as an element of a multiplicative matrix group. We have to control these noncommutative multiplicative structures in linear function spaces and to establish their convergence to the corresponding linear additive structures. In order to give some details in this direction we cannot avoid introducing some minimal notation.

Finite-strain elastoplasticity is usually based on the multiplicative decomposition $\nabla \varphi=F_{\mathrm{el}} F_{\mathrm{pl}}$ [Lee69]. Here $\varphi: \Omega \rightarrow \mathbb{R}^{d}$ is the deformation of the body with respect to the reference configuration $\Omega \subset \mathbb{R}^{d}(d=2,3)$ while $F_{\mathrm{el}}, F_{\mathrm{pl}} \in \mathrm{SL}(d)$ stand for the elastic strain and the plastic strain, respectively. Then, the energy stored in the body is written as

$$
\int_{\Omega} W_{\mathrm{el}}\left(\nabla \varphi F_{\mathrm{pl}}^{-1}\right) d x+\int_{\Omega} W_{\mathrm{h}}\left(F_{\mathrm{pl}}\right) d x
$$

where $W_{\mathrm{el}}$ is a frame-indifferent elastic stored-energy density and $W_{\mathrm{h}}$ describes hardening. The plastic flow rule is expressed by means of a suitably defined dissipation distance $D: \operatorname{SL}(d) \times \operatorname{SL}(d) \rightarrow[0, \infty]$. In particular $D\left(F_{\mathrm{pl}}, \widehat{F}_{\mathrm{pl}}\right)$ represents the minimal dissipated energy for an evolution from the plastic strain $F_{\mathrm{pl}}$ to $\widehat{F}_{\mathrm{pl}}$ and is given by means of a positively 1 -homogeneous dissipation function $R$ by

$$
D\left(F_{\mathrm{pl}}, \widehat{F}_{\mathrm{pl}}\right)=D\left(I, \widehat{F}_{\mathrm{pl}} F_{\mathrm{pl}}^{-1}\right)=\inf \int_{\Omega} \int_{0}^{1} R\left(\dot{P} P^{-1}\right) d t d x
$$

the infimum being taken over all smooth trajectories $P:[0,1] \rightarrow \mathbb{R}^{d \times d}$ connecting $F_{\mathrm{pl}}$ to $\widehat{F}_{\mathrm{pl}}$. Starting from these functionals, by specifying loadings, and boundary and initial conditions, suitably weak solutions of the quasi-static finite-plasticity system (see Section 2) can be defined. We refer to [Mie03] for more information on the mathematical modeling of finite-strain elastoplasticity. There also models with additional hardening variables are given. Here we refrain from maximal generality in order to emphasize the main features of the limiting process.

Let now the deformation and the plastic strain be small. In particular, for $\varepsilon>0$ let $\varphi_{\varepsilon}=\mathrm{id}+\varepsilon u$ and $F_{\mathrm{pl}, \varepsilon}=I+\varepsilon z$ where $u$ is interpreted as the displacement and $z$ is the linearized plastic strain. Correspondingly, we have $F_{\mathrm{el}, \varepsilon}=\nabla \varphi_{\varepsilon} F_{\mathrm{pl}, \varepsilon}^{-1}=(\mathrm{id}+$ $\varepsilon \nabla u)(I+\varepsilon z)^{-1}$ and we are led to consider of the rescaled finite-strain elastoplasticity energy and dissipation functionals

$$
\frac{1}{\varepsilon^{2}} \int_{\Omega} W_{\mathrm{el}}\left((I+\varepsilon \nabla u)(I+\varepsilon z)^{-1}\right) d x+\frac{1}{\varepsilon^{2}} \int_{\Omega} W_{\mathrm{h}}(I+\varepsilon z) d x, \quad \frac{1}{\varepsilon} D(I+\varepsilon z, I+\varepsilon \widehat{z}) .
$$


Note that the rescalings above are such that, by assuming that $W_{\mathrm{el}}$ and $W_{\mathrm{h}}$ admit a quadratic expansion around identity, one can check that

$$
\begin{aligned}
\frac{1}{\varepsilon^{2}} \int_{\Omega} W_{\mathrm{el}}\left((I+\varepsilon \nabla u)(I+\varepsilon z)^{-1}\right) d x & \rightarrow \frac{1}{2} \int_{\Omega}(\nabla u-z): \mathbb{C}(\nabla u-z) d x, \\
\frac{1}{\varepsilon^{2}} \int_{\Omega} W_{\mathrm{h}}(I+\varepsilon z) d x & \rightarrow \frac{1}{2} \int_{\Omega} z: \mathbb{H} z d x \\
\frac{1}{\varepsilon} D(I+\varepsilon z, I+\varepsilon \widehat{z}) & \rightarrow \int_{\Omega} R(\widehat{z}-z) d x .
\end{aligned}
$$

This pointwise convergence is the classical justification of linearization in plasticity. On the other hand, it is not sufficient alone for proving that finite-strain elastoplasticity trajectories actually converge to a solution of the linearized-plasticity system.

Before going on let us mention that the solution concept which is here under consideration is that of energetic solutions. Starting from [MT04], this notion has been extensively applied in many different rate-independent contexts. We record, however, that one of the main motivations for introducing energetic solutions was exactly that of targeting existence theories for finite-strain elastoplasticity. In this respect, note that the only available existence result for finite-strain elastoplastic evolution has been recently obtained within the energetic solvability framework in [MM09] after adding the regularizing term $\left|\nabla F_{\mathrm{pl}}\right|^{r}$ for $r>1$ (see also [MM06] for some preliminary result),

Our result consists in proving the convergence of energetic solutions of the finitestrain elastoplasticity system to linearized-plasticity solutions. In order to prove this convergence we follow the abstract evolutionary $\Gamma$-convergence theory for energetic solutions of rate-independent processes developed in [MRS08]. We mention that this evolutionary $\Gamma$-convergence method has recently attracted attention and has been successfully considered in connection with numerical approximations [KMR05, MR09, GP06a], damage [BRM09, TM10], fracture [GP06b], delamination [RSZ09], dimension reduction [FPZ10, LM11], homogenization [Tim09], and optimal control [Rin08, Rin09].

According to [MRS08], the convergence of the trajectories $\left(u_{\varepsilon}, z_{\varepsilon}\right)$ follows by proving two separate $\Gamma$-liminf inequalities for energy and dissipation, and constructing a mutual recovery sequence relating both. Note that separate $\Gamma$-convergence for energy and dissipation is not sufficient to pass to the limit within rate-independent processes. Apart from the additional technicalities due to the presence of the plastic strain and the dissipation functional, it is the delicate construction of the mutual recovery sequence that distinguishes our argument from all the already developed stationary analyses in the spirit of [DNP02].

\section{Problem setup and results}

Let the reference configuration $\Omega \subset \mathbb{R}^{d}$ be an open, bounded, and connected set with Lipschitz boundary. Moreover, let $\Gamma \subset \partial \Omega$ be relatively open with $\mathcal{H}^{d-1}(\Gamma)>0$. We define the state space as

$$
\mathcal{Q}:=\mathcal{U} \times \mathcal{Z}:=\left\{u \in \mathrm{H}^{1}\left(\Omega ; \mathbb{R}^{d}\right) \mid u=0 \text { on } \Gamma\right\} \times \mathrm{L}^{2}\left(\Omega ; \mathbb{R}^{d \times d}\right) .
$$


Note that the choice of the homogeneous Dirichlet condition on the displacement $u$ is just for simplicity. In particular, different boundary conditions may be considered as well.

For each given $A \in \mathbb{R}^{d \times d}$ we denote its symmetric and antisymmetric parts as $A^{\text {sym }}:=\left(A+A^{\top}\right) / 2$ and $A^{\text {anti }}=A-A^{\text {sym }}$. We indicate by $\mathbb{R}_{\text {sym }}^{d \times d}$ and $\mathbb{R}_{\text {anti }}^{d \times d}$ the subspaces of symmetric and antisymmetric tensors, respectively, whereas $\mathbb{R}_{\mathrm{dev}}^{d \times d}$ stands for the subspace of symmetric and trace-free tensors, also called deviatoric tensors. The standard Euclidian tensor norm is denoted by $|\cdot|$ and, for all $A \in \mathbb{R}^{d \times d}$ and $\tau>0, B_{\tau}(A)$ indicates the ball $\left\{B \in \mathbb{R}^{d \times d}|| A-B \mid<\tau\right\}$. Moreover, $|\cdot| \mathbb{T}$ stands for the seminorm

$$
|A|_{\mathbb{T}}^{2}:=\frac{1}{2} A: \mathbb{T} A
$$

where the 4-tensor $\mathbb{T} \in \mathbb{R}^{d \times d \times d \times d}$ is symmetric $\left(\mathbb{T}_{i j k \ell}=\mathbb{T}_{k \ell i j}\right)$ and positive semidefinite. For finite-strain elastoplasticity we use the classical notations

$$
\begin{aligned}
\mathrm{SL}(d) & :=\left\{P \in \mathbb{R}^{d \times d} \mid \operatorname{det} P=1\right\}, \\
\mathrm{SO}(d) & :=\left\{R \in \mathrm{SL}(d) \mid R^{\top} R=R R^{\top}=I\right\}, \\
\mathrm{GL}_{+}(d) & :=\left\{Q \in \mathbb{R}^{d \times d} \mid \operatorname{det} Q>0\right\} .
\end{aligned}
$$

We assume that the elastic energy density functional $W_{\mathrm{el}}$ satisfies

$$
\begin{aligned}
& W_{\mathrm{el}}: \mathbb{R}^{d \times d} \rightarrow[0, \infty], \quad W_{\mathrm{el}} \in \mathrm{C}^{1}\left(\mathrm{GL}_{+}(d)\right), \quad W_{\mathrm{el}} \equiv \infty \text { on } \mathbb{R}^{d \times d} \backslash \mathrm{GL}_{+}(d), \\
& \forall F \in \mathrm{GL}_{+}(d) \forall R \in \mathrm{SO}(d): \quad W_{\mathrm{el}}(R F)=W_{\mathrm{el}}(F), \\
& \forall F \in \mathrm{GL}_{+}(d): \quad W_{\mathrm{el}}(F) \geq c_{1} \operatorname{dist}^{2}(F, \mathrm{SO}(d)), \\
& \forall F \in \mathrm{GL}_{+}(d): \quad\left|F^{\top} \partial_{F} W_{\mathrm{el}}(F)\right| \leq c_{2}\left(W_{\mathrm{el}}(F)+1\right), \\
& \exists \mathbb{C} \geq 0 \forall \delta>0 \exists c_{\mathrm{el}}(\delta)>0 \forall A \in B_{c_{\mathrm{el}}(\delta)}(0):\left.\left.\quad\left|W_{\mathrm{el}}(I+A)-\right| A\right|_{\mathbb{C}} ^{2}|\leq \delta| A\right|_{\mathbb{C}} ^{2},
\end{aligned}
$$

for some positive $c_{1}, c_{2}$. Assumption (2.1b) is nothing but frame indifference, and the nondegeneracy requirement (2.1c) is quite classical. Assumption (2.1d) entails the controllability of the Mandel tensor $F^{\top} \partial_{F} W_{\mathrm{el}}(F)$ by means of the energy. This is a crucial condition in finite-strain elastoplasticity (cf. [Bal84b, Bal02]) and was used in the context of rate-independent processes in [FM06, MM09]. Condition (2.1e) encodes the local quadratic character of $W_{\mathrm{el}}$ around the identity. More precisely, (2.1e) states that $|\cdot|_{\mathbb{C}}$ is the second order Taylor expansion of $W_{\mathrm{el}}$ at $I$, and may be reformulated by saying that $A \mapsto W_{\mathrm{el}}(I+A)$ is locally restrained between two multiples of $|\cdot|_{\mathbb{C}}^{2}$, namely,

$$
\forall \delta>0 \forall A \in B_{c_{\mathrm{el}}(\delta)}(0): \quad(1-\delta)|A|_{\mathbb{C}}^{2} \leq W_{\mathrm{el}}(I+A) \leq(1+\delta)|A|_{\mathbb{C}}^{2} .
$$

Moreover, (2.1e) entails

$$
W_{\mathrm{el}}(I)=0, \quad \partial_{F} W_{\mathrm{el}}(I)=0, \quad \partial_{F}^{2} W_{\mathrm{el}}(I)=\mathbb{C},
$$

which, in particular, implies that the reference state is stress free. On the other hand, by assuming (2.2) and letting $W_{\mathrm{el}} \in \mathrm{C}^{2}$ in a neighborhood of $I$, relation (2.1e) follows. 
Note that the symmetry of the elastic tensor $\mathbb{C}$ (implicitly assumed in the notation $|\cdot| \mathbb{C})$ may be directly obtained from the last condition of (2.2) by assuming additional smoothness of $W_{\mathrm{el}}$. Moreover, letting $A \in \mathbb{R}^{d \times d}$ be given, as we have $\exp \left(A^{\mathrm{anti}}\right) \in$ $\mathrm{SO}(d)$, the frame indifference (2.1b) entails that the function $t \mapsto \partial_{F} W_{\mathrm{el}}\left(\exp \left(t A^{\mathrm{anti}}\right)\right)$ is constantly equal to $\partial_{F} W_{\mathrm{el}}(I)=0$. Hence, by taking its derivative with respect to $t$ and evaluating it at $t=0$ we get $\mathbb{C} A^{\text {anti }}=0$. Thus, $\mathbb{C}$ necessarily also has the so called minor symmetries $\mathbb{C}_{i j k \ell}=\mathbb{C}_{j i k \ell}=\mathbb{C}_{i j \ell k}$ and we have

$$
\forall A \in \mathbb{R}^{d \times d}: \quad \mathbb{C} A=\mathbb{C} A^{\mathrm{sym}} .
$$

On the other hand, as a consequence of the nondegeneracy (2.1c) and assumption (2.1e) we deduce that $\mathbb{C}$ is positive definite on $\mathbb{R}_{\mathrm{sym}}^{d \times d}$. Indeed, by linearizing $d(\cdot, \mathrm{SO}(d)$ ) around identity we have [FJM02, (3.21)]

$$
\forall B \in \mathbb{R}^{d \times d}: \quad d(B, \mathrm{SO}(d))=\left|B^{\mathrm{sym}}-I\right|+O\left(|B-I|^{2}\right) .
$$

Hence, given $A \in \mathbb{R}^{d \times d}$ and $\eta, \delta>0$, by choosing $B=I+\eta A$ above we have

$$
c_{1}\left|A^{\mathrm{sym}}\right|^{2} \stackrel{(2.4)}{=} \lim _{\eta \rightarrow 0} \frac{c_{1}}{\eta^{2}} d^{2}(I+\eta A, \operatorname{SO}(d)) \stackrel{(2.1 \mathrm{c})}{\leq} \lim _{\eta \rightarrow 0} \frac{1}{\eta^{2}} W_{\mathrm{el}}(I+\eta A) \stackrel{(2.1 \mathrm{e})}{\leq}(1+\delta)|A|_{\mathbb{C}}^{2},
$$

so that, by taking $\delta \rightarrow 0$, we have

$$
\forall A \in \mathbb{R}^{d \times d}: \quad c_{1}\left|A^{\mathrm{sym}}\right|^{2} \leq|A|_{\mathbb{C}}^{2}=\left|A^{\mathrm{sym}}\right|_{\mathbb{C}}^{2} .
$$

Note that all assumptions (2.1a)-(2.1e) are consistent with the usual polyconvexity framework

$$
F \mapsto W_{\mathrm{el}}(F) \quad \text { polyconvex, } \quad W_{\mathrm{el}}(F) \rightarrow \infty \quad \text { as } \operatorname{det} F \rightarrow 0 .
$$

Our assumptions on the hardening functional $W_{\mathrm{h}}: \mathbb{R}^{d \times d} \rightarrow[0, \infty] \mathrm{read}$

$$
W_{\mathrm{h}}(P):= \begin{cases}\widetilde{W}_{\mathrm{h}}(P) & \text { if } P \in K \\ \infty & \text { if } P \in \mathbb{R}^{d \times d} \backslash K\end{cases}
$$

$K$ is compact in $\operatorname{SL}(d)$ and contains a neighborhood of $I$,

$\widetilde{W}_{\mathrm{h}}: \mathbb{R}^{d \times d} \rightarrow \mathbb{R}$ is locally Lipschitz continuous,

$$
\begin{aligned}
& \exists \mathbb{H} \geq 0 \forall \delta>0 \exists c_{\mathrm{h}}(\delta)>0 \forall A \in B_{c_{\mathrm{h}}(\delta)}(0):\left.\left.\quad\left|\tilde{W}_{\mathrm{h}}(I+A)-\right| A\right|_{\mathbb{H}} ^{2}|\leq \delta| A\right|_{\mathbb{H}} ^{2}, \\
& \exists c_{3}>0 \forall A \in \mathbb{R}^{d \times d}: \quad W_{\mathrm{h}}(I+A) \geq c_{3}|A|^{2} .
\end{aligned}
$$

Note that by assumption (2.6b) we can find a constant $c_{K}>0$ such that

$$
\begin{aligned}
& P \in K \Rightarrow|P|+\left|P^{-1}\right| \leq c_{K}, \\
& P \in \operatorname{SL}(d) \backslash K \Rightarrow|P-I| \geq 1 / c_{K} .
\end{aligned}
$$

The rather strong technical assumption on $W_{\mathrm{h}}$ that its effective domain $K=\{P \in \mathrm{SL}(d) \mid$ $\left.W_{\mathrm{h}}(P)<\infty\right\}$ satisfies $(2.7)$ is crucial as it will provide $\mathrm{L}^{\infty}$-bounds that are essential in 
order to control the multiplicative terms $(I+\varepsilon \nabla u)(I+\varepsilon z)^{-1}$. Moreover, by combining (2.6d) and (2.6e) we check that

$$
\forall A \in \mathbb{R}^{d \times d}: \quad c_{3}|A|^{2} \leq|A|_{\mathbb{H}}^{2} .
$$

As for the dissipation we assume that

$$
\begin{aligned}
& R^{\mathrm{dev}}: \mathbb{R}_{\operatorname{dev}}^{d \times d} \rightarrow[0, \infty) \text { is convex and positively 1-homogeneous, } \\
& \forall P \in \mathbb{R}_{\mathrm{dev}}^{d \times d}: \quad c_{4}|P| \leq R^{\operatorname{dev}}(P) \leq c_{5}|P|, \\
& R: \mathbb{R}^{d \times d} \rightarrow[0, \infty], \quad R(z):= \begin{cases}R^{\operatorname{dev}}(z) & \text { if } z \in \mathbb{R}_{\operatorname{dev}}^{d \times d} \\
\infty & \text { else, }\end{cases}
\end{aligned}
$$

for positive $c_{4}, c_{5}$. Moreover, we define

$D: \mathbb{R}^{d \times d} \times \mathbb{R}^{d \times d} \rightarrow[0, \infty], \quad$ with $D(P, \widehat{P})=D\left(I, \widehat{P} P^{-1}\right)$ given by

$D(I, \widehat{P}):=\inf \left\{\int_{0}^{1} R\left(\dot{P} P^{-1}\right) d t \mid P \in \mathrm{C}^{1}\left(0,1 ; \mathbb{R}^{d \times d}\right), P(0)=I, P(1)=\widehat{P}\right\}$.

If $P$ is not invertible, we set $D(P, \widehat{P})=\infty$. Note in particular that $D(I, P)<\infty$ implies $\operatorname{det} P=1$. Moreover, there exists $c_{6}>0$ such that

$$
\forall P, Q \in K \subset \operatorname{SL}(d): \quad D(P, Q) \leq c_{6}, \quad D(I, P) \leq c_{6}|P-I| .
$$

For the first estimate the continuity of $D$ and the compactness of $K$ are sufficient. For the second, we need to establish the estimate only for $P$ close to $I$, where it follows from $D(I, P) \leq R^{\operatorname{dev}}(\log P) \leq c_{5}|\log P| \leq c_{6}|P-I|$, since the matrix logarithm is welldefined and Lipschitz continuous in a neighborhood of $I$. See also [MM09, Ex. 3.2] and the references given there for global bounds on $D$.

The quasistatic evolution of the finite-strain and linearized elastoplasticity systems are driven by the energy functionals $\mathcal{W}_{\varepsilon}, \mathcal{W}_{0}: \mathcal{Q} \rightarrow(-\infty, \infty]$ given by

$$
\begin{aligned}
& \mathcal{W}_{\varepsilon}(u, z):=\frac{1}{\varepsilon^{2}} \int_{\Omega} W_{\mathrm{el}}\left((I+\varepsilon \nabla u)(I+\varepsilon z)^{-1}\right) d x+\frac{1}{\varepsilon^{2}} \int_{\Omega} W_{\mathrm{h}}(I+\varepsilon z) d x, \\
& \mathcal{W}_{0}(u, z):=\int_{\Omega}\left|\nabla u^{\mathrm{sym}}-z^{\mathrm{sym}}\right|_{\mathbb{C}}^{2} d x+\int_{\Omega}|z|_{\mathbb{H}}^{2} d x .
\end{aligned}
$$

Note that if the second integral in the definition of $\mathcal{W}_{\varepsilon}(u, z)$ is finite, then $I+\varepsilon z \in K$ almost everywhere by (2.6a). Hence, the inverse $(I+\varepsilon z)^{-1}$ exists and the first integral is well defined.

We prescribe the generalized loading as

$$
\ell \in W^{1,1}\left(0, T ; \mathcal{U}^{\prime}\right)
$$


and, by letting $\ell_{\varepsilon}:=\varepsilon \ell$, we introduce some notation for the total energy functionals $\mathcal{E}_{\varepsilon}, \mathcal{E}_{0}:[0, T] \times \mathcal{Q} \rightarrow(-\infty, \infty]$ as

$$
\begin{aligned}
& \mathcal{E}_{\varepsilon}(t, u, z):=\mathcal{W}_{\varepsilon}(u, z)-\frac{1}{\varepsilon}\left\langle\ell_{\varepsilon}(t), u\right\rangle=\mathcal{W}_{\varepsilon}(u, z)-\langle\ell(t), u\rangle, \\
& \mathcal{E}_{0}(t, u, z):=\mathcal{W}_{0}(u, z)-\langle\ell(t), u\rangle .
\end{aligned}
$$

Finally, the dissipative character of the evolution is encoded into the dissipation functions $D_{\varepsilon}, D_{0}: \mathbb{R}^{d \times d} \times \mathbb{R}^{d \times d} \rightarrow[0, \infty]$ and functionals $\mathcal{D}_{\varepsilon}, \mathcal{D}_{0}:\left(L^{1}\left(\Omega ; \mathbb{R}^{d \times d}\right)\right)^{2} \rightarrow[0, \infty]$ given by

$$
\begin{aligned}
& D_{\varepsilon}\left(z_{1}, z_{2}\right):=\frac{1}{\varepsilon} D\left(I+\varepsilon z_{1}, I+\varepsilon z_{2}\right), \quad D_{0}\left(z_{1}, z_{2}\right):=R\left(z_{2}-z_{1}\right), \\
& \mathcal{D}_{\varepsilon}\left(z_{1}, z_{2}\right):=\int_{\Omega} D_{\varepsilon}\left(z_{1}, z_{2}\right) d x, \quad \mathcal{D}_{0}\left(z_{1}, z_{2}\right):=\int_{\Omega} D_{0}\left(z_{1}, z_{2}\right) d x .
\end{aligned}
$$

The total dissipation of the process over the time interval $[0, t] \subset[0, T]$ will be given by

$$
\operatorname{Diss}_{\mathcal{D}_{\varepsilon}}(z ;[0, t]):=\sup \left\{\sum_{i=1}^{N} \mathcal{D}_{\varepsilon}\left(z\left(t^{i}\right), z\left(t^{i-1}\right)\right) \mid 0=t^{0}<\cdots<t^{N}=t\right\}
$$

where the sup is taken over all partitions of $[0, t]$.

We define a Rate-Independent System (RIS) to be the triple $\left(\mathcal{Q}, \mathcal{E}_{\varepsilon}, \mathcal{D}_{\varepsilon}\right)$ given by the choice of the state space $\mathcal{Q}$ and the energy and dissipation functionals $\mathcal{E}_{\varepsilon}$ and $\mathcal{D}_{\varepsilon}$. The term evolutionary $\Gamma$-convergence refers to a suitable notion of convergence for rateindependent systems in the spirit of [MRS08], which in particular entails the convergence of the respective energetic solutions.

A crucial structure in the energetic formulation of RIS is the set $\mathcal{S}_{\varepsilon}(t)$ of stable states at time $t \in[0, T]$, which is defined to be

$$
\begin{aligned}
& \mathcal{S}_{\varepsilon}(t):=\left\{(u, z) \in \mathcal{Q} \mid \mathcal{E}_{\varepsilon}(t, u, z)<\infty\right. \text { and } \\
&\left.\qquad \mathcal{E}_{\varepsilon}(t, u, z) \leq \mathcal{E}_{\varepsilon}(t, \widehat{u}, \widehat{z})+\mathcal{D}_{\varepsilon}(z, \hat{z}) \forall(\widehat{u}, \widehat{z}) \in \mathcal{Q}\right\} .
\end{aligned}
$$

Our assumption on the initial data reads

$$
\begin{aligned}
& \mathcal{S}_{\varepsilon}(0) \ni\left(u_{\varepsilon}^{0}, z_{\varepsilon}^{0}\right) \rightarrow\left(u_{0}^{0}, z_{0}^{0}\right) \quad \text { weakly in } \mathcal{Q}, z_{0}^{0} \in \mathrm{L}^{2}\left(\Omega ; \mathbb{R}_{\mathrm{dev}}^{d \times d}\right), \\
& \mathcal{E}_{\varepsilon}\left(0, u_{\varepsilon}^{0}, z_{\varepsilon}^{0}\right) \rightarrow \mathcal{E}_{0}\left(0, u_{0}^{0}, z_{0}^{0}\right) .
\end{aligned}
$$

Note that the latter assumption is not empty as it is satisfied at least by the natural choice $\left(u_{0}, z_{0}\right)=(0,0)$ if $\ell(0)=0$.

Definition 2.1 (Energetic solutions). Let $\varepsilon \geq 0$. We say that a trajectory $q_{\varepsilon}:[0, T] \rightarrow$ $\left(u_{\varepsilon}, z_{\varepsilon}\right) \in \mathcal{Q}$ is an energetic solution (related to the $\left.\operatorname{RIS}\left(\mathcal{Q}, \mathcal{E}_{\varepsilon}, \mathcal{D}_{\varepsilon}\right)\right)$ if $\left(u_{\varepsilon}(0), z_{\varepsilon}(0)\right)=$ $\left(u_{\varepsilon}^{0}, z_{\varepsilon}^{0}\right)$, the map $t \mapsto\left\langle\dot{\ell}, u_{\varepsilon}\right\rangle$ is integrable, and, for all $t \in[0, T]$,

$$
\begin{aligned}
& \left(u_{\varepsilon}(t), z_{\varepsilon}(t)\right) \in \mathcal{S}_{\varepsilon}(t) \\
& \mathcal{E}_{\varepsilon}\left(t, u_{\varepsilon}(t), z_{\varepsilon}(t)\right)+\operatorname{Diss}_{\mathcal{D}_{\varepsilon}}\left(z_{\varepsilon} ;[0, t]\right)=\mathcal{E}_{\varepsilon}\left(0, u_{\varepsilon}^{0}, z_{\varepsilon}^{0}\right)-\int_{0}^{t}\left\langle\dot{\ell}, u_{\varepsilon}\right\rangle d s .
\end{aligned}
$$


An energetic solution will be called a finite-plasticity solution if $\varepsilon>0$, and a linearizedplasticity solution for $\varepsilon=0$.

Note that linearized-plasticity solutions $\left(u_{0}, z_{0}\right)$ are unique in view of the quadratic and uniformly convex character of $\mathcal{W}_{0}$. Moreover, from assumption (2.13) we find that $\left(u_{0}, z_{0}\right) \in \mathrm{W}^{1,1}(0, T ; \mathcal{Q})$ and

$$
\forall t \in[0, T]: \quad \operatorname{Diss}_{\mathcal{D}_{0}}\left(z_{0} ;[0, t]\right)=\int_{0}^{t} R\left(\dot{z}_{0}\right) d s .
$$

The reader is referred to [Hil50, Lub90, Mar75] for a general introduction to plasticity and to [HR99, Joh76, Suq81] for the classical well-posedness theory for linearized elastoplasticity.

Our main result reads as follows and will be proved in Section 3 as a special instance of the general theory of [MRS08].

Theorem 2.2 (Finite plasticity $\Gamma$-converges to linearized plasticity). Assume (2.1)(2.6), (2.10), and (2.13)-(2.14). Let $\left(u_{\varepsilon}, z_{\varepsilon}\right)$ be a finite-plasticity solution. Then $\left(u_{\varepsilon}(t), z_{\varepsilon}(t)\right) \rightarrow\left(u_{0}(t), z_{0}(t)\right)$ weakly in $\mathcal{Q}$ for all $t \in[0, T]$ where $\left(u_{0}, z_{0}\right)$ is the unique linearized-plasticity solution.

Theorem 2.2 is exclusively a convergence result. In particular, we assume that finiteplasticity solutions exist. Note however that the existence of finite-plasticity solutions is presently not known within our minimal assumptions. A possibility here would be to consider directly some more regular situations including extra compactifying terms like $\left|\nabla F_{\mathrm{pl}}\right|^{r}(r>1)$ ensuring that finite-plasticity solutions exist [MM09]. We shall not follow this line here but rather present a second result based on approximate minimizers of the related incremental problems. Indeed, given time partitions $0=t_{\varepsilon}^{i}<\cdots<t_{\varepsilon}^{N_{\varepsilon}}=T$ with diameters $\tau_{\varepsilon}:=\max _{i=1, \ldots, N_{\varepsilon}}\left(t_{\varepsilon}^{i}-t_{\varepsilon}^{i-1}\right) \rightarrow 0$ as $\varepsilon \rightarrow 0$, the (iterative) incremental problem

$$
\left(u_{\varepsilon}^{i}, z_{\varepsilon}^{i}\right) \in \underset{(u, v) \in \mathcal{Q}}{\operatorname{Arg} \min }\left(\mathcal{E}_{\varepsilon}\left(t_{\varepsilon}^{i}, u, z\right)+\mathcal{D}_{\varepsilon}\left(z_{\varepsilon}^{i-1}, z\right)\right) \quad \text { for } i=1, \ldots, N_{\varepsilon}
$$

may not be solvable (cf. [CHM02]; see also [Mie04, MM06] for some additional discussion). Hence, following [MRS08, Sec. 4] we fix a sequence $0<\alpha_{\varepsilon} \rightarrow 0$ in order to control the tolerances for the minimizations and consider the following approximate incremental problem:

Find iteratively $\left(u_{\varepsilon}^{i}, z_{\varepsilon}^{i}\right) \in \mathcal{Q}$ such that

$$
\mathcal{E}_{\varepsilon}\left(t_{\varepsilon}^{i}, u_{\varepsilon}^{i}, z_{\varepsilon}^{i}\right)+\mathcal{D}_{\varepsilon}\left(z_{\varepsilon}^{i-1}, z_{\varepsilon}^{i}\right) \leq\left(t_{\varepsilon}^{i}-t_{\varepsilon}^{i-1}\right) \alpha_{\varepsilon}+\inf _{(u, v) \in \mathcal{Q}}\left(\mathcal{E}_{\varepsilon}\left(t_{\varepsilon}^{i}, u, z\right)+\mathcal{D}_{\varepsilon}\left(z_{\varepsilon}^{i-1}, z\right)\right) .
$$

By the definition of infimum the latter always admits solutions and we will show the following convergence result. 
Theorem 2.3 (Convergence of approximate incremental minimizers). Under the assumptions of Theorem 2.2 let $\left(u_{\varepsilon}^{i}, z_{\varepsilon}^{i}\right)$ be approximate incremental minimizers and $\left(\bar{u}_{\varepsilon}, \bar{z}_{\varepsilon}\right)$ be the corresponding right-continuous, piecewise-constant interpolants on the time partitions. Then $\left(\bar{u}_{\varepsilon}(t), \bar{z}_{\varepsilon}(t)\right) \rightarrow\left(u_{0}(t), z_{0}(t)\right)$ weakly in $\mathcal{Q}$ for all $t \in[0, T]$ where $\left(u_{0}, z_{0}\right)$ is the unique linearized-plasticity solution.

In the finite-elasticity case (stationary), using ideas from [DNP02] the convergence of approximate minimizers has been considered in [PT09].

\section{Proofs}

The argument basically follows the lines of the abstract analysis of [MRS08]. Still, our setting cannot be completely recovered from the application of the above-mentioned abstract theory as extra care is needed for the treatment of multiplicative nonlinearities. We hence provide here an independent proof. After establishing the coercivity of the energy in Subsection 3.1, the proof strategy relies on providing two separate $\Gamma$-liminf inequalities for $\mathcal{E}_{\varepsilon}$ and $\mathcal{D}_{\varepsilon}$ and a mutual recovery sequence argument relating both. This is done in Subsections 3.2 and 3.3 below. Finally, the proofs of Theorems 2.2 and 2.3 are outlined in Subsections 3.4 and 3.5, respectively.

A caveat on notation: henceforth the symbol $c$ stands for any positive constant independent of $\varepsilon$ and $\delta$ but possibly depending on the fixed data. In particular, note that $c$ may change from line to line. Moreover, in the following we use the short-hand notation, for all $A \in \mathbb{R}^{d \times d}$,

$W_{\mathrm{el}}^{\varepsilon}(A):=\frac{1}{\varepsilon^{2}} W_{\mathrm{el}}(I+\varepsilon A), \quad W_{\mathrm{h}}^{\varepsilon}(A):=\frac{1}{\varepsilon^{2}} W_{\mathrm{h}}(I+\varepsilon A), \quad \widetilde{W}_{\mathrm{h}}^{\varepsilon}(A):=\frac{1}{\varepsilon^{2}} \widetilde{W}_{\mathrm{h}}(I+\varepsilon A)$.

\subsection{Energy coercivity}

We start by providing a uniform coercivity result for the energy. It follows the ideas in [DNP02] and relies on the Rigidity Lemma [FJM02, Thm. 3.1].

Lemma 3.1 (Coercivity). There exists $c>0$ such that, for all $(u, z) \in \mathcal{Q}$,

$$
\|\nabla u\|_{\mathrm{L}^{2}}^{2}+\|z\|_{\mathrm{L}^{2}}^{2}+\|\varepsilon z\|_{\mathrm{L}^{\infty}}^{2} \leq c\left(1+\mathcal{W}_{\varepsilon}(u, z)\right) .
$$

Proof. Let us assume with no loss of generality that $\mathcal{W}_{\varepsilon}(u, z)<\infty$, so that $I+\varepsilon z \in K$ almost everywhere by assumption (2.6a). Hence, $|I+\varepsilon z| \leq c_{K}$ almost everywhere from property (2.7), and the inverse $(I+\varepsilon z)^{-1}$ exists almost everywhere. Thus, we have $\|\varepsilon z\|_{L^{\infty}} \leq c$. Moreover, one readily checks from the coercivity (2.6e) that

$$
c_{3}\|z\|_{\mathrm{L}^{2}}^{2} \leq \int_{\Omega} W_{\mathrm{h}}^{\varepsilon}(z) d x \leq \mathcal{W}_{\varepsilon}(u, z) .
$$


For the displacement $u$ we follow ideas from [DNP02]. Given any $Q \in \mathrm{SO}(d)$ by letting $\varphi=\mathrm{id}+\varepsilon u$ and $F_{\mathrm{el}}=\nabla \varphi(I+\varepsilon z)^{-1}$ we have

$$
\begin{aligned}
|\nabla \varphi-Q|^{2} & =|\nabla \varphi-Q(I+\varepsilon z)+\varepsilon Q z|^{2}=\left|\left(F_{\mathrm{el}}-Q\right)(I+\varepsilon z)+\varepsilon Q z\right|^{2} \\
& \leq c\left(\left|F_{\mathrm{el}}-Q\right|^{2}|I+\varepsilon z|^{2}+\varepsilon^{2}|z|^{2}\right) \leq c\left(\left|F_{\mathrm{el}}-Q\right|^{2}+\varepsilon^{2}|z|^{2}\right) .
\end{aligned}
$$

In particular, by passing to the infimum over $Q \in \mathrm{SO}(d)$ we conclude that

$$
\operatorname{dist}^{2}(\nabla \varphi, \mathrm{SO}(d)) \leq c\left(\operatorname{dist}^{2}\left(F_{\mathrm{el}}, \mathrm{SO}(d)\right)+\varepsilon^{2}|z|^{2}\right) .
$$

By taking the integral in space and using the nondegeneracy condition (2.1c) we obtain

$\int_{\Omega} \operatorname{dist}^{2}(\nabla \varphi, \mathrm{SO}(d)) d x \leq c \int_{\Omega} \operatorname{dist}^{2}\left(F_{\mathrm{el}}, \mathrm{SO}(d)\right) d x+c \varepsilon^{2} \int_{\Omega}|z|^{2} d x \stackrel{(3.2)}{\leq} \varepsilon^{2} c \mathcal{W}_{\varepsilon}(u, z)$.

Hence, the Rigidity Lemma [FJM02, Thm. 3.1] ensures that

$$
\|\nabla \varphi-\widehat{Q}\|_{\mathrm{L}^{2}}^{2} \leq \varepsilon^{2} c \mathcal{W}_{\varepsilon}(u, z)
$$

for some constant rotation $\widehat{Q} \in \mathrm{SO}(d)$. Finally, using [DNP02, Prop. 3.4] and $\left.\varphi\right|_{\Gamma}=\mathrm{id}$ as $u \in \mathcal{U}$, we conclude $|\widehat{Q}-I|^{2} \leq \varepsilon^{2} c \mathcal{W}_{\varepsilon}(u, z)$. Therefore

$$
\|\nabla u\|_{\mathrm{L}^{2}}^{2}=\frac{1}{\varepsilon^{2}}\|\nabla \varphi-I\|_{\mathrm{L}^{2}}^{2} \leq \frac{2}{\varepsilon^{2}}\|\nabla \varphi-\widehat{Q}\|_{\mathrm{L}^{2}}^{2}+\frac{2}{\varepsilon^{2}}\|\widehat{Q}-I\|_{\mathrm{L}^{2}}^{2} \leq c \mathcal{W}_{\varepsilon}(u, z),
$$

and the bound (3.1) follows.

\section{2. $\Gamma$-liminf inequalities}

Next, we turn our attention to the proof of the separate $\Gamma$-liminf inequalities for energy and dissipation. Let us start with a statement concerning the energy densities.

Lemma 3.2. Under assumptions (2.1e) and (2.6d), we have

$$
W_{\mathrm{el}}^{\varepsilon} \rightarrow|\cdot|_{\mathbb{C}}^{2} \text { and } \tilde{W}_{\mathrm{h}}^{\varepsilon} \rightarrow|\cdot|_{\mathbb{H}}^{2} \quad \text { locally uniformly. }
$$

Moreover,

$$
|z|_{\mathbb{H}}^{2} \leq \inf \left\{\liminf _{\varepsilon \rightarrow 0} W_{\mathrm{h}}^{\varepsilon}\left(z_{\varepsilon}\right) \mid z_{\varepsilon} \rightarrow z\right\}
$$

Proof. Let $K_{0} \Subset \mathbb{R}^{d \times d}$, fix $\delta>0$ and find the corresponding $c_{\mathrm{el}}(\delta)>0$ from condition (2.1e). As $\varepsilon K_{0} \subset B_{c_{\mathrm{el}}(\delta)}(0)$ for $\varepsilon$ sufficiently small, we have

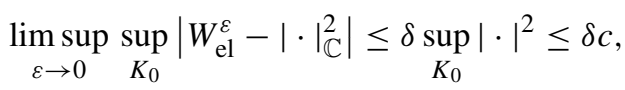

and local uniform convergence follows from $\delta>0$ being arbitrary. The same argument applies to $\widetilde{W}_{\mathrm{h}}^{\varepsilon}$.

As for the $\Gamma$-liminf inequality (3.4), let $z_{\varepsilon} \rightarrow z$ and assume with no loss of generality that $\sup _{\varepsilon} W_{\mathrm{h}}^{\varepsilon}\left(z_{\varepsilon}\right)<\infty$. Hence, $W_{\mathrm{h}}^{\varepsilon}\left(z_{\varepsilon}\right)=\widetilde{W}_{\mathrm{h}}^{\varepsilon}\left(z_{\varepsilon}\right)$ and the inequality follows from the uniform convergence proved above.

We are now in a position to prove the $\Gamma$-liminf estimate for the energy. It follows indeed from (3.3) and the lower-semicontinuity result of Lemma 4.2 below. 
Lemma 3.3 ( $\Gamma$-liminf for energy). For all $(u, z) \in \mathcal{Q}$ we have

$$
\mathcal{W}_{0}(u, z) \leq \inf \left\{\liminf _{\varepsilon \rightarrow 0} \mathcal{W}_{\varepsilon}\left(u_{\varepsilon}, z_{\varepsilon}\right) \mid\left(u_{\varepsilon}, z_{\varepsilon}\right) \rightarrow(u, z) \text { weakly in } \mathcal{Q}\right\} .
$$

Proof. Let $\left(u_{\varepsilon}, z_{\varepsilon}\right) \rightarrow(u, z)$ weakly in $\mathcal{Q}$. We can assume with no loss of generality that $\sup _{\varepsilon} \mathcal{W}_{\varepsilon}\left(u_{\varepsilon}, z_{\varepsilon}\right)<\infty$. Owing to the $\Gamma$-liminf inequality (3.4) and the lowersemicontinuity Lemma 4.2 we readily conclude that

$$
\int_{\Omega}|z|_{\mathbb{H}}^{2} \leq \liminf _{\varepsilon \rightarrow 0} \int_{\Omega} W_{\mathrm{h}}^{\varepsilon}\left(z_{\varepsilon}\right) d x=\liminf _{\varepsilon \rightarrow 0} \frac{1}{\varepsilon^{2}} \int_{\Omega} W_{\mathrm{h}}\left(I+\varepsilon z_{\varepsilon}\right) d x .
$$

Moreover, $\mathcal{W}_{\varepsilon}\left(u_{\varepsilon}, z_{\varepsilon}\right)<\infty$ implies $\varepsilon z_{\varepsilon} \in K-I$ almost everywhere. In particular, $\varepsilon z_{\varepsilon}$ are bounded in $\mathrm{L}^{\infty}$. The same holds for $\left(I+\varepsilon z_{\varepsilon}\right)^{-1}$ as

$$
\left(I+\varepsilon z_{\varepsilon}\right)^{-1}=\operatorname{cof}\left(I+\varepsilon z_{\varepsilon}\right) / \operatorname{det}\left(I+\varepsilon z_{\varepsilon}\right)=\operatorname{cof}\left(I+\varepsilon z_{\varepsilon}\right) .
$$

We define the auxiliary tensors

$$
w_{\varepsilon}:=\frac{1}{\varepsilon}\left(\left(I+\varepsilon z_{\varepsilon}\right)^{-1}-I+\varepsilon z_{\varepsilon}\right)=\varepsilon\left(I+\varepsilon z_{\varepsilon}\right)^{-1} z_{\varepsilon}^{2},
$$

so that $\left(I+\varepsilon z_{\varepsilon}\right)^{-1}=I-\varepsilon z_{\varepsilon}+\varepsilon w_{\varepsilon}$. By the first equality in (3.6) we have $\left\|\varepsilon w_{\varepsilon}\right\|_{L^{\infty}} \leq c$, while the second gives

$$
\left\|w_{\varepsilon}\right\|_{\mathrm{L}^{1}}=\varepsilon\left\|\left(I+\varepsilon z_{\varepsilon}\right)^{-1} z_{\varepsilon}^{2}\right\|_{\mathrm{L}^{1}} \leq c \varepsilon\left\|z_{\varepsilon}\right\|_{\mathrm{L}^{2}}^{2} \leq c \varepsilon
$$

where we have also used the boundedness in $\mathrm{L}^{2}$ of $z_{\varepsilon}$ from (3.1). Thus, by interpolation, $w_{\varepsilon}$ is bounded in $\mathrm{L}^{2}$ as well, so that $w_{\varepsilon} \rightarrow 0$ weakly in $\mathrm{L}^{2}$.

Given $A_{\varepsilon}:=\left(F_{\mathrm{el}, \varepsilon}-I\right) / \varepsilon$ we want to show the weak $\mathrm{L}^{2}$ convergence $A_{\varepsilon} \rightarrow \nabla u-z$. From

$$
A_{\varepsilon}=\frac{1}{\varepsilon}\left(\left(I+\varepsilon \nabla u_{\varepsilon}\right)\left(I+\varepsilon z_{\varepsilon}\right)^{-1}-I\right)
$$

we find $I+\varepsilon A_{\varepsilon}=\left(I+\varepsilon \nabla u_{\varepsilon}\right)\left(I+\varepsilon z_{\varepsilon}\right)^{-1}$ and compute that

$$
A_{\varepsilon}=\frac{1}{\varepsilon}\left(\left(I+\varepsilon \nabla u_{\varepsilon}\right)\left(I-\varepsilon z_{\varepsilon}+\varepsilon w_{\varepsilon}\right)-I\right)=\nabla u_{\varepsilon}-z_{\varepsilon}+w_{\varepsilon}-\varepsilon\left(\nabla u_{\varepsilon} z_{\varepsilon}-\nabla u_{\varepsilon} w_{\varepsilon}\right) .
$$

Hence, as $\nabla u_{\varepsilon}-z_{\varepsilon} \rightarrow \nabla u-z$ and $w_{\varepsilon} \rightarrow 0$ weakly in $\mathrm{L}^{2}$, we have to show $v_{\varepsilon}:=$ $\nabla u_{\varepsilon}\left(\varepsilon z_{\varepsilon}-\varepsilon w_{\varepsilon}\right) \rightarrow 0$ weakly in $\mathrm{L}^{2}$ as well. Indeed, the boundedness in $\mathrm{L}^{2}$ of $v_{\varepsilon}$ follows from $\left\|\nabla u_{\varepsilon}\right\|_{\mathrm{L}^{2}} \leq c$ (see (3.1)) and the $\mathrm{L}^{\infty}$ boundedness of $\varepsilon z_{\varepsilon}$ and $\varepsilon w_{\varepsilon}$. Moreover, since $z_{\varepsilon}$ and $w_{\varepsilon}$ are bounded in $\mathrm{L}^{2}$ we have $\left\|v_{\varepsilon}\right\|_{\mathrm{L}^{1}} \leq c \varepsilon$ and conclude $v_{\varepsilon} \rightarrow 0$ weakly in $\mathrm{L}^{2}$.

Eventually, owing to Lemma 3.2, we can exploit the lower-semicontinuity Lemma 4.2 to obtain

$\int_{\Omega}|\nabla u-z|_{\mathbb{C}}^{2} \leq \liminf _{\varepsilon \rightarrow 0} \int_{\Omega} W_{\mathrm{el}}^{\varepsilon}\left(A_{\varepsilon}\right) d x=\liminf _{\varepsilon \rightarrow 0} \frac{1}{\varepsilon^{2}} \int_{\Omega} W_{\mathrm{el}}\left(\left(I+\varepsilon \nabla u_{\varepsilon}\right)\left(I+\varepsilon z_{\varepsilon}\right)^{-1}\right) d x$.

Finally, by recalling relation (2.3) and the already established (3.5), the assertion follows.

Before moving to the $\Gamma$-liminf inequality for the dissipation functionals $\mathcal{D}_{\varepsilon}$, we prepare here a preliminary result on the functions $D_{\varepsilon}$. 
Lemma 3.4 ( $\Gamma$-convergence of $\left.D_{\varepsilon}\right) . D_{\varepsilon} \rightarrow D_{0}$ in the sense of $\Gamma$-convergence.

Proof. $\Gamma$-liminf inequality. Let $\left(z_{\varepsilon}, \widehat{z}_{\varepsilon}\right) \rightarrow(z, \widehat{z})$ and assume with no loss of generality that $\sup _{\varepsilon} D_{\varepsilon}\left(z_{\varepsilon}, \widehat{z}_{\varepsilon}\right)<\infty$. In particular, $\left(I+\varepsilon \widehat{z}_{\varepsilon}\right)\left(I+\varepsilon z_{\varepsilon}\right)^{-1} \in \operatorname{SL}(d)$. By defining

$$
\zeta_{\varepsilon}:=\frac{1}{\varepsilon}\left(\left(I+\varepsilon \widehat{z}_{\varepsilon}\right)\left(I+\varepsilon z_{\varepsilon}\right)^{-1}-I\right)=\widehat{z}_{\varepsilon}-z_{\varepsilon}+w_{\varepsilon}-\varepsilon \widehat{z}_{\varepsilon} z_{\varepsilon}+\varepsilon \widehat{z}_{\varepsilon} w_{\varepsilon}
$$

where $w_{\varepsilon}$ is given in (3.6), we readily check that $I+\varepsilon \zeta_{\varepsilon} \in \operatorname{SL}(d)$ and $\zeta_{\varepsilon} \rightarrow \widehat{z}-z$.

Let now $t \mapsto P_{\varepsilon}(t) \in \mathrm{C}^{1}\left(0,1 ; \mathbb{R}^{d \times d}\right)$ be such that $P_{\varepsilon}(0)=I, P_{\varepsilon}(1)=I+\varepsilon \zeta_{\varepsilon}$, and

$$
D\left(I, I+\varepsilon \zeta_{\varepsilon}\right) \geq(1-\varepsilon) \int_{0}^{1} R\left(\dot{P}_{\varepsilon} P_{\varepsilon}^{-1}\right) d t .
$$

Such a function $P_{\varepsilon}$ exists by the very definition of $D$. By possibly reparametrizing $P_{\varepsilon}$ and using assumption (2.10b) and bound (2.12) we can assume that

$$
c_{4}\left|\dot{P}_{\varepsilon}(t) P_{\varepsilon}^{-1}(t)\right| \stackrel{(2.10 \mathrm{~b})}{\leq} R\left(\dot{P}_{\varepsilon}(t) P_{\varepsilon}^{-1}(t)\right) \leq 2 D\left(I, I+\varepsilon \zeta_{\varepsilon}\right) \stackrel{(2.12)}{\leq} c \varepsilon .
$$

Hence,

$$
\left|P_{\varepsilon}(t)-I\right| \leq \int_{0}^{t}\left|\dot{P}_{\varepsilon} P_{\varepsilon}^{-1}\right|\left|P_{\varepsilon}\right| d s \leq c \varepsilon \int_{0}^{t}\left|P_{\varepsilon}\right| d s \leq c \varepsilon\left(1+\int_{0}^{t}\left|P_{\varepsilon}-I\right| d s\right)
$$

so that $P_{\varepsilon} \rightarrow I$ uniformly by the Gronwall Lemma.

By defining $\widehat{P}_{\varepsilon}(t)=I+\left(P_{\varepsilon}(t)-I\right) / \varepsilon$ one has $\widehat{P}_{\varepsilon}(0)=I$ and $\widehat{P}_{\varepsilon}(1)=I+\zeta_{\varepsilon}$. Moreover, as $\varepsilon \widehat{P}_{\varepsilon}=\dot{P}_{\varepsilon}$ and $R$ is positively 1-homogeneous (2.10a), we have

$$
\frac{1}{\varepsilon} D\left(I, I+\varepsilon \zeta_{\varepsilon}\right) \geq(1-\varepsilon) \int_{0}^{1} R\left(\widehat{\widehat{P}}_{\varepsilon} P_{\varepsilon}^{-1}\right) d t .
$$

Owing now to bound (3.8), by possibly extracting not relabeled subsequences, we deduce that $\stackrel{\widehat{P}_{\varepsilon}}{\rightarrow} Q$ weakly-star in $\mathrm{L}^{\infty}\left(0,1 ; \mathbb{R}^{d \times d}\right)$ and

$$
\begin{aligned}
\liminf _{\varepsilon \rightarrow 0} D_{\varepsilon}\left(z_{\varepsilon}, \widehat{z}_{\varepsilon}\right) & =\liminf _{\varepsilon \rightarrow 0} \frac{1}{\varepsilon} D\left(I, I+\varepsilon \zeta_{\varepsilon}\right) \geq \liminf _{\varepsilon \rightarrow 0} \int_{0}^{1} R\left(\hat{P}_{\varepsilon} P_{\varepsilon}^{-1}\right) d t \geq \int_{0}^{1} R(Q) d t \\
& \geq R(\tilde{Q})
\end{aligned}
$$

where we have exploited the lower-semicontinuity tool of Lemma 4.2 and Jensen's inequality with $\tilde{Q}=\int_{0}^{1} Q d t$.

Finally, by integrating we have

$$
\tilde{Q}=\int_{0}^{1} Q d t=\lim _{\varepsilon \rightarrow 0} \int_{0}^{1} \widehat{P_{\varepsilon}} d t=\lim _{\varepsilon \rightarrow 0} \zeta_{\varepsilon}=\widehat{z}-z
$$

so that we have checked

$$
\liminf _{\varepsilon \rightarrow 0} D_{\varepsilon}\left(z_{\varepsilon}, \widehat{z}_{\varepsilon}\right) \geq R(\widehat{z}-z)
$$


Recovery sequence. Given $\zeta \in \mathbb{R}_{\operatorname{dev}}^{d \times d}$ we have $\exp (\zeta) \in \operatorname{SL}(d)$ and, by taking $P(t):=$ $\exp (t \zeta)$ in the definition of $D$, we readily check that $D(I, \exp (\zeta)) \leq R(\zeta)$.

Let now $z, \widehat{z} \in \mathbb{R}_{\mathrm{dev}}^{d \times d}$ be given and define

$$
\widehat{z}_{\varepsilon}=\frac{1}{\varepsilon}(\exp (\varepsilon(\widehat{z}-z))(I+\varepsilon z)-I) .
$$

As $\left(I+\varepsilon \widehat{z}_{\varepsilon}\right)(I+\varepsilon z)^{-1}=\exp (\varepsilon(\widehat{z}-z))$, we have

$$
\limsup _{\varepsilon \rightarrow 0} D_{\varepsilon}\left(z, \widehat{z}_{\varepsilon}\right)=\limsup _{\varepsilon \rightarrow 0} \frac{1}{\varepsilon} D(I, \exp (\varepsilon(\widehat{z}-z))) \leq R(\widehat{z}-z)=D_{0}(z, \widehat{z})
$$

so that $\left(z, \widehat{z}_{\varepsilon}\right)$ is a recovery sequence.

Owing to Lemma 3.4, it suffices now to apply the lower-semicontinuity result in Lemma 4.2 in order to establish the $\Gamma$-liminf inequality for the dissipation functionals. More precisely, we have the following.

Lemma 3.5 ( $\Gamma$-liminf for dissipation).

$$
\mathcal{D}_{0}(z, \widehat{z}) \leq \inf \left\{\liminf _{\varepsilon \rightarrow 0} \mathcal{D}_{\varepsilon}\left(z_{\varepsilon}, \widehat{z}_{\varepsilon}\right) \mid\left(z_{\varepsilon}, \widehat{z}_{\varepsilon}\right) \rightarrow(z, \widehat{z}) \text { weakly in }\left(\mathrm{L}^{2}\left(\Omega ; \mathbb{R}^{d \times d}\right)\right)^{2}\right\} .
$$

\subsection{Mutual recovery sequence}

We now come to the construction of a mutual recovery sequence. Let us recall from [MRS08] that indeed two separate $\Gamma$-limsup inequalities for energy and dissipation generally do not suffice for passing to the limit in RIS. In particular, the construction of recovery sequences for energy and dissipation has to be mutually coordinated.

Lemma 3.6 (Mutual recovery sequence). Let $t \in[0, T],\left(u_{\varepsilon}, z_{\varepsilon}\right) \rightarrow\left(u_{0}, z_{0}\right)$ weakly in $\mathcal{Q}$, and

$$
\sup _{\varepsilon} \mathcal{E}_{\varepsilon}\left(t, u_{\varepsilon}, z_{\varepsilon}\right)<\infty
$$

Moreover, let $\left(\widehat{u}_{0}, \widehat{z}_{0}\right):=\left(u_{0}, z_{0}\right)+(\tilde{u}, \tilde{z})$ with $(\tilde{u}, \tilde{z}) \in \mathrm{C}_{\mathrm{c}}^{\infty}\left(\Omega ; \mathbb{R}^{d}\right) \times \mathrm{C}_{\mathrm{c}}^{\infty}\left(\Omega ; \mathbb{R}_{\mathrm{dev}}^{d \times d}\right)$. Then there exist $\left(\widehat{u}_{\varepsilon}, \widehat{z}_{\varepsilon}\right) \in \mathcal{Q}$ such that $\left(\widehat{u}_{\varepsilon}, \widehat{z}_{\varepsilon}\right) \rightarrow\left(\widehat{u}_{0}, \widehat{z}_{0}\right)$ weakly in $\mathcal{Q}$ and

$$
\begin{aligned}
\limsup _{\varepsilon \rightarrow 0}\left(\mathcal{E}_{\varepsilon}\left(t, \widehat{u}_{\varepsilon}, \widehat{z}_{\varepsilon}\right)-\mathcal{E}_{\varepsilon}(t,\right. & \left.\left.u_{\varepsilon}, z_{\varepsilon}\right)+\mathcal{D}_{\varepsilon}\left(z_{\varepsilon}, \widehat{z}_{\varepsilon}\right)\right) \\
& \leq \mathcal{E}_{0}\left(t, \widehat{u}_{0}, \widehat{z}_{0}\right)-\mathcal{E}_{0}\left(t, u_{0}, z_{0}\right)+\mathcal{D}_{0}\left(z_{0}, \widehat{z}_{0}\right) .
\end{aligned}
$$

Proof. For the sake of clarity, we decompose the argument into several steps. The general strategy of the proof is to choose $\left(\widehat{u}_{\varepsilon}, \widehat{z}_{\varepsilon}\right)$ and show convergence to $\left(\widehat{u}_{0}, \widehat{z}_{0}\right)$,

$$
\limsup _{\varepsilon \rightarrow 0} \mathcal{D}_{\varepsilon}\left(z_{\varepsilon}, \widehat{z}_{\varepsilon}\right) \leq \mathcal{D}_{0}\left(z_{0}, \widehat{z}_{0}\right)=R(\tilde{z}),
$$


and

$$
\limsup _{\varepsilon \rightarrow 0}\left(\mathcal{E}_{\varepsilon}\left(t, \widehat{u}_{\varepsilon}, \widehat{z}_{\varepsilon}\right)-\mathcal{E}_{\varepsilon}\left(t, u_{\varepsilon}, z_{\varepsilon}\right)\right) \leq \mathcal{E}_{0}\left(t, \widehat{u}_{0}, \widehat{z}_{0}\right)-\mathcal{E}_{0}\left(t, u_{0}, z_{0}\right) .
$$

Note that in order to establish the latter we cannot argue on individual terms but rather aim at exploiting certain cancellations. This resembles the situation of the so-called quadratic trick (see, e.g., [MT05]) and crucially uses (2.1d) as well as the smoothness of ( $\tilde{u}, \tilde{z})$. In particular, note that within this proof the constant $c$ may depend on $\tilde{u}$ and $\tilde{z}$ as well.

Step 1: Choice of the mutual recovery sequence. By defining the functions $\psi_{\varepsilon}:=\mathrm{id}+\varepsilon \tilde{u}$ and $\varphi_{\varepsilon}:=\mathrm{id}+\varepsilon u_{\varepsilon}$ and the set

$$
\Omega_{\varepsilon}:=\left\{x \in \Omega \mid \exp (\varepsilon \tilde{z}(x))\left(I+\varepsilon z_{\varepsilon}(x)\right) \in K\right\},
$$

the proof of the lemma follows by checking that the choices

$$
\begin{aligned}
& \widehat{u}_{\varepsilon}:=\frac{1}{\varepsilon}\left(\psi_{\varepsilon} \circ \varphi_{\varepsilon}-\mathrm{id}\right), \\
& \widehat{z}_{\varepsilon}:= \begin{cases}\frac{1}{\varepsilon}\left(\exp (\varepsilon \tilde{z})\left(I+\varepsilon z_{\varepsilon}\right)-I\right) & \text { on } \Omega_{\varepsilon}, \\
z_{\varepsilon} & \text { else, }\end{cases}
\end{aligned}
$$

satisfy (3.11) and, in particular, $\left(\widehat{u}_{\varepsilon}, \widehat{z}_{\varepsilon}\right) \rightarrow\left(\widehat{u}_{0}, \widehat{z}_{0}\right)$ weakly in $\mathcal{Q}$. The construction of $\widehat{u}_{\varepsilon}$ via a composition and of $\widehat{z}_{\varepsilon}$ via matrix exponential and multiplication is necessary in order to deal with the multiplicative nature of finite-strain elastoplasticity.

Note that the construction of the mutual recovery sequence is compatible with the constraint $\operatorname{det}\left(I+\varepsilon \nabla \widehat{u}_{\varepsilon}\right)>0$ considered in (2.1a). Indeed, by letting $\varepsilon$ be small enough we see that $I+\varepsilon \nabla \tilde{u}$ is everywhere positive definite, hence $\operatorname{det}(I+\varepsilon \nabla \tilde{u})>0$. In particular, as $\operatorname{det}\left(I+\varepsilon \nabla u_{\varepsilon}\right)>0$ almost everywhere by (2.1a) and (3.10), we have

$$
\operatorname{det}\left(I+\varepsilon \nabla \widehat{u}_{\varepsilon}\right)=\operatorname{det}\left(\nabla \psi_{\varepsilon}\left(\varphi_{\varepsilon}\right) \nabla \varphi_{\varepsilon}\right)=\operatorname{det}\left(I+\varepsilon \nabla \tilde{u}\left(\varphi_{\varepsilon}\right)\right) \operatorname{det}\left(I+\varepsilon \nabla u_{\varepsilon}\right)>0
$$

almost everywhere as well. That is, $I+\varepsilon \nabla \widehat{u}_{\varepsilon} \in \mathrm{GL}_{+}(d)$ almost everywhere.

From the bound (3.10) we readily see that $I+\varepsilon z_{\varepsilon} \in \mathrm{SL}(d)$ almost everywhere. Hence, upon noting that

$$
I+\varepsilon \widehat{z}_{\varepsilon}= \begin{cases}\exp (\varepsilon \tilde{z})\left(I+\varepsilon z_{\varepsilon}\right) & \text { on } \Omega_{\varepsilon} \\ I+\varepsilon z_{\varepsilon} & \text { else }\end{cases}
$$

we immediately check that $I+\varepsilon \widehat{z}_{\varepsilon} \in K \subset \mathrm{SL}(d)$ almost everywhere and is bounded in $\mathrm{L}^{\infty}$. Using the fact that $\operatorname{tr} \tilde{z}=0$ we have $\operatorname{det} \exp (\varepsilon \tilde{z})=\exp (\varepsilon \operatorname{tr} \tilde{z})=1$ and hence $\exp (\varepsilon \tilde{z})\left(I+\varepsilon z_{\varepsilon}\right) \in \operatorname{SL}(d)$ almost everywhere.

Next, note that the measure of the complement of $\Omega_{\varepsilon}$ can be controlled by means of a Chebyshev estimate. Indeed, (2.8) gives

$$
\begin{aligned}
\left|\Omega \backslash \Omega_{\varepsilon}\right| & =\int_{\Omega \backslash \Omega_{\varepsilon}} 1 d x \leq c_{K}^{2} \int_{\Omega}\left|\exp (\varepsilon \tilde{z})\left(I+\varepsilon z_{\varepsilon}\right)-I\right|^{2} d x \\
& =c_{K}^{2} \int_{\Omega}\left|\exp (\varepsilon \tilde{z})-I+\varepsilon \exp (\varepsilon \tilde{z}) z_{\varepsilon}\right|^{2} d x \leq c \varepsilon^{2}\left(1+\int_{\Omega} z_{\varepsilon}^{2} d x\right) \leq c \varepsilon^{2} .
\end{aligned}
$$


Now, one finds that

$$
\begin{aligned}
& \widehat{z}_{\varepsilon}-z_{\varepsilon}=\frac{1}{\varepsilon}\left(\exp (\varepsilon \tilde{z})\left(I+\varepsilon z_{\varepsilon}\right)-I\right)-z_{\varepsilon}=\frac{1}{\varepsilon}(\exp (\varepsilon \tilde{z})-I)\left(I+\varepsilon z_{\varepsilon}\right) \quad \text { on } \Omega_{\varepsilon}, \\
& \widehat{z}_{\varepsilon}-z_{\varepsilon}=0 \quad \text { on } \Omega \backslash \Omega_{\varepsilon},
\end{aligned}
$$

$\left|\Omega \backslash \Omega_{\varepsilon}\right| \rightarrow 0$, and $\widehat{z}_{\varepsilon}$ and $z_{\varepsilon}$ are bounded in $\mathrm{L}^{2}$. Hence, we readily check that

$$
\widehat{z}_{\varepsilon}-z_{\varepsilon} \rightarrow \tilde{z} \quad \text { strongly in } \mathrm{L}^{2}\left(\Omega ; \mathbb{R}^{d \times d}\right) .
$$

This implies that $\widehat{z}_{\varepsilon} \rightarrow \widehat{z}_{0}=z_{0}+\tilde{z}$ weakly in $\mathrm{L}^{2}$, hence

$$
\widehat{z}_{\varepsilon}+z_{\varepsilon} \rightarrow \widehat{z}_{0}+z_{0} \quad \text { weakly in } \mathrm{L}^{2}\left(\Omega ; \mathbb{R}^{d \times d}\right) .
$$

From the energy bound (3.10) and the coercivity Lemma 3.1 we infer that $u_{\varepsilon}$ is bounded in $\mathrm{H}^{1}$ and $\varepsilon u_{\varepsilon} \rightarrow 0$ strongly in $\mathrm{L}^{2}$. Hence, $\left\|\varphi_{\varepsilon}-\mathrm{id}\right\|_{\mathrm{L}^{2}}=\varepsilon\left\|u_{\varepsilon}\right\|_{\mathrm{L}^{2}} \leq c \varepsilon$ and, by the Lipschitz continuity of $\nabla \tilde{u}$, we conclude that

$$
\left\|\nabla \tilde{u}\left(\varphi_{\varepsilon}\right)-\nabla \tilde{u}\right\|_{\mathrm{L}^{2}} \leq c\left\|\varphi_{\varepsilon}-\mathrm{id}\right\|_{\mathrm{L}^{2}}=c \varepsilon\left\|u_{\varepsilon}\right\|_{\mathrm{L}^{2}} \leq c \varepsilon .
$$

Moreover, by computing

$$
\begin{aligned}
\nabla \widehat{u}_{\varepsilon} & =\frac{1}{\varepsilon}\left(\nabla \psi_{\varepsilon}\left(\varphi_{\varepsilon}\right) \nabla \varphi_{\varepsilon}-I\right)=\frac{1}{\varepsilon}\left((I+\varepsilon \nabla \tilde{u})\left(\varphi_{\varepsilon}\right) \nabla \varphi_{\varepsilon}-I\right) \\
& =\frac{1}{\varepsilon}\left(\nabla \varphi_{\varepsilon}+\varepsilon \nabla \tilde{u}\left(\varphi_{\varepsilon}\right) \nabla \varphi_{\varepsilon}-I\right)=\nabla u_{\varepsilon}+\nabla \tilde{u}\left(\varphi_{\varepsilon}\right)+\varepsilon \nabla \tilde{u}\left(\varphi_{\varepsilon}\right) \nabla u_{\varepsilon}
\end{aligned}
$$

we obtain

$$
\begin{aligned}
\left\|\left(\nabla \widehat{u}_{\varepsilon}-\nabla u_{\varepsilon}\right)-\nabla \tilde{u}\right\|_{\mathrm{L}^{2}} & \leq\left\|\nabla \tilde{u}\left(\varphi_{\varepsilon}\right)-\nabla \tilde{u}\right\|_{\mathrm{L}^{2}}+\left\|\varepsilon \nabla \tilde{u}\left(\varphi_{\varepsilon}\right) \nabla u_{\varepsilon}\right\|_{\mathrm{L}^{2}} \\
& \stackrel{(3.14)}{\leq} c \varepsilon+c \varepsilon\left\|\nabla u_{\varepsilon}\right\|_{\mathrm{L}^{2}} \leq c \varepsilon,
\end{aligned}
$$

and this implies that $\widehat{u}_{\varepsilon} \rightarrow \widehat{u}_{0}=u_{0}+\tilde{u}$ weakly in $\mathrm{H}^{1}$.

The tensors $A_{\varepsilon}=\left(F_{\mathrm{el}, \varepsilon}-I\right) / \varepsilon$ and $\widehat{A}_{\varepsilon}=\left(\widehat{F}_{\mathrm{el}, \varepsilon}-I\right) / \varepsilon$ satisfy

$$
A_{\varepsilon}=\frac{1}{\varepsilon}\left(\left(I+\varepsilon \nabla u_{\varepsilon}\right)\left(I+\varepsilon z_{\varepsilon}\right)^{-1}-I\right), \quad \widehat{A}_{\varepsilon}=\frac{1}{\varepsilon}\left(\left(I+\varepsilon \nabla \widehat{u}_{\varepsilon}\right)\left(I+\varepsilon \widehat{z}_{\varepsilon}\right)^{-1}-I\right)
$$

and hence are both bounded in $\mathrm{L}^{2}$ by (2.7).

Fix now $\delta$ and let $c_{\mathrm{el}}(\delta)$ and $c_{\mathrm{h}}(\delta)$ be given by conditions (2.1e) and (2.6d), respectively. For all $\varepsilon>0$ we define the sets

$$
\begin{aligned}
U_{\varepsilon}^{\delta} & :=\left\{x \in \Omega|| \varepsilon A_{\varepsilon}(x)|+| \varepsilon \widehat{A}_{\varepsilon}(x) \mid \leq c_{\mathrm{el}}(\delta)\right\}, \\
Z_{\varepsilon}^{\delta} & :=\left\{x \in \Omega|| \varepsilon z_{\varepsilon}(x)|+| \varepsilon \widehat{z}_{\varepsilon}(x) \mid \leq c_{\mathrm{h}}(\delta)\right\} .
\end{aligned}
$$

We refer to the latter as good sets, because strains are there under control and we can replace the nonlinear densities $W_{\mathrm{el}}$ and $W_{\mathrm{h}}$ by their quadratic expansions via (2.1e) and (2.6d). In particular, on the good sets the quadratic character of the expansions will entail 
the control of the difference of the energy contributions by means of a suitable cancellation (quadratic trick). On the other hand, we term bad sets the corresponding complements $\Omega \backslash U_{\varepsilon}^{\delta}$ and $\Omega \backslash Z_{\varepsilon}^{\delta}$ where the quadratic expansions are a priori not available. Using some nontrivial cancellations, we will show that the difference of the energy contributions on the bad sets is infinitesimal. Note preliminarily that the integrands on the bad sets blow up while the bad sets have small measure. Indeed,

$$
\begin{aligned}
& \left|\Omega \backslash U_{\varepsilon}^{\delta}\right|=\int_{\Omega \backslash U_{\varepsilon}^{\delta}} 1 d x \leq \frac{\varepsilon^{2}}{c_{\mathrm{el}}^{2}(\delta)} \int_{\Omega}\left(\left|A_{\varepsilon}\right|+\left|\widehat{A}_{\varepsilon}\right|\right)^{2} d x \leq \frac{c \varepsilon^{2}}{c_{\mathrm{el}}^{2}(\delta)}, \\
& \left|\Omega \backslash Z_{\varepsilon}^{\delta}\right|=\int_{\Omega \backslash Z_{\varepsilon}^{\delta}} 1 d x \leq \frac{\varepsilon^{2}}{c_{\mathrm{h}}^{2}(\delta)} \int_{\Omega}\left(\left|z_{\varepsilon}\right|+\left|\widehat{z}_{\varepsilon}\right|\right)^{2} d x \leq \frac{c \varepsilon^{2}}{c_{\mathrm{h}}^{2}(\delta)} .
\end{aligned}
$$

Step 2: Treatment of the dissipation term. As $\widehat{z}_{\varepsilon}=z_{\varepsilon}$ on $\Omega \backslash \Omega_{\varepsilon}$, one has

$$
\mathcal{D}_{\varepsilon}\left(z_{\varepsilon}, \widehat{z}_{\varepsilon}\right)=\frac{1}{\varepsilon} \int_{\Omega_{\varepsilon}} D(I, \exp (\varepsilon \tilde{z})) d x \leq \frac{1}{\varepsilon} \int_{\Omega} D(I, \exp (\varepsilon \tilde{z})) d x .
$$

In the construction of the recovery sequence in the proof of Lemma 3.4 we have proved that

$$
\limsup _{\varepsilon \rightarrow 0} \frac{1}{\varepsilon} D(I, \exp (\varepsilon \tilde{z})) \leq R(\tilde{z}) .
$$

Finally, by taking the lim sup in (3.18) and using (3.19) we conclude that

$$
\begin{aligned}
\limsup _{\varepsilon \rightarrow 0} \mathcal{D}_{\varepsilon}\left(z_{\varepsilon}, \widehat{z}_{\varepsilon}\right) & =\limsup _{\varepsilon \rightarrow 0} \frac{1}{\varepsilon} \int_{\Omega} D(I, \exp (\varepsilon \tilde{z})) d x \leq \int_{\Omega} R(\tilde{z}) d x \\
& =\mathcal{D}_{0}\left(z_{0}, \widehat{z}_{0}\right) .
\end{aligned}
$$

Step 3: Limsup for the differences of the elastic energy terms. Let us start by rewriting the tensors $A_{\varepsilon}$ as

$$
A_{\varepsilon}=\nabla u_{\varepsilon}-z_{\varepsilon}+w_{\varepsilon}-\varepsilon \nabla u_{\varepsilon} z_{\varepsilon}+\varepsilon \nabla u_{\varepsilon} w_{\varepsilon}
$$

where $w_{\varepsilon}$ is given by (3.6). On the other hand, we have

$$
\begin{aligned}
\widehat{A}_{\varepsilon}= & \frac{1}{\varepsilon}\left(\left(I+\varepsilon \nabla \widehat{u}_{\varepsilon}\right)\left(I-\varepsilon z_{\varepsilon}+\varepsilon w_{\varepsilon}\right) \exp (-\varepsilon \tilde{z})-I\right) \\
= & \left(\nabla \widehat{u}_{\varepsilon}-z_{\varepsilon}+w_{\varepsilon}-\varepsilon \nabla \widehat{u}_{\varepsilon} z_{\varepsilon}+\varepsilon \nabla \widehat{u}_{\varepsilon} w_{\varepsilon}\right) \exp (-\varepsilon \tilde{z}) \\
& +\frac{1}{\varepsilon}(\exp (-\varepsilon \tilde{z})-I) \quad \text { on } \Omega_{\varepsilon}, \\
\widehat{A}_{\varepsilon}= & \frac{1}{\varepsilon}\left(\left(I+\varepsilon \nabla \widehat{u}_{\varepsilon}\right)\left(I-\varepsilon z_{\varepsilon}+\varepsilon w_{\varepsilon}\right)-I\right) \\
= & \nabla \widehat{u}_{\varepsilon}-z_{\varepsilon}+w_{\varepsilon}-\varepsilon \nabla \widehat{u}_{\varepsilon} z_{\varepsilon}+\varepsilon \nabla \widehat{u}_{\varepsilon} w_{\varepsilon} \quad \text { on } \Omega \backslash \Omega_{\varepsilon} .
\end{aligned}
$$


Hence, one can compute that

$$
\begin{aligned}
\widehat{A}_{\varepsilon}-A_{\varepsilon}= & \left(\nabla \widehat{u}_{\varepsilon}-\nabla u_{\varepsilon}\right)\left(I-\varepsilon z_{\varepsilon}+\varepsilon w_{\varepsilon}\right)+\frac{1}{\varepsilon}(\exp (-\varepsilon \tilde{z})-I), \\
& +\left(\nabla \widehat{u}_{\varepsilon}-z_{\varepsilon}+w_{\varepsilon}-\varepsilon \nabla \widehat{u}_{\varepsilon} z_{\varepsilon}+\varepsilon \nabla \widehat{u}_{\varepsilon} w_{\varepsilon}\right)(\exp (-\varepsilon \tilde{z})-I) \quad \text { on } \Omega_{\varepsilon}, \\
\widehat{A}_{\varepsilon}-A_{\varepsilon}= & \left(\nabla \widehat{u}_{\varepsilon}-\nabla u_{\varepsilon}\right)\left(I-\varepsilon z_{\varepsilon}+\varepsilon w_{\varepsilon}\right) \quad \text { on } \Omega \backslash \Omega_{\varepsilon} .
\end{aligned}
$$

In particular, owing to convergence (3.15) and the $\mathrm{L}^{\infty}$ bounds for $\varepsilon z_{\varepsilon}$ and $\varepsilon w_{\varepsilon}$ (see the discussion after (3.6)) we see that $\left(\nabla \widehat{u}_{\varepsilon}-\nabla u_{\varepsilon}\right)\left(I-\varepsilon z_{\varepsilon}+\varepsilon w_{\varepsilon}\right)$ converges to $\nabla \tilde{u}$ strongly in $\mathrm{L}^{2}$. Thus, by recalling that $w_{\varepsilon} \rightarrow 0$ weakly in $\mathrm{L}^{2}$ it is a standard matter to check that

$$
\begin{aligned}
& \widehat{A}_{\varepsilon}+A_{\varepsilon} \rightarrow\left(\nabla \widehat{u}_{0}-\widehat{z}_{0}\right)+\left(\nabla u_{0}-z_{0}\right) \quad \text { weakly in } \mathrm{L}^{2}\left(\Omega ; \mathbb{R}^{d \times d}\right), \\
& \widehat{A}_{\varepsilon}-A_{\varepsilon} \rightarrow \nabla \tilde{u}-\tilde{z} \quad \text { strongly in } \mathrm{L}^{2}\left(\Omega ; \mathbb{R}^{d \times d}\right) .
\end{aligned}
$$

On the good set $U_{\varepsilon}^{\delta}$ we will use the assumption (2.1e) to deduce that

$$
\begin{aligned}
W_{\mathrm{el}}^{\varepsilon}\left(\widehat{A}_{\varepsilon}\right)-W_{\mathrm{el}}^{\varepsilon}\left(A_{\varepsilon}\right) & \leq\left|\widehat{A}_{\varepsilon}\right|_{\mathbb{C}}^{2}-\left|A_{\varepsilon}\right|_{\mathbb{C}}^{2}+\delta\left(\left|\widehat{A}_{\varepsilon}\right|_{\mathbb{C}}^{2}+\left|A_{\varepsilon}\right|_{\mathbb{C}}^{2}\right) \\
& =\frac{1}{2}\left(\widehat{A}_{\varepsilon}-A_{\varepsilon}\right): \mathbb{C}\left(\widehat{A}_{\varepsilon}+A_{\varepsilon}\right)+\delta\left(\left|\widehat{A}_{\varepsilon}\right|_{\mathbb{C}}^{2}+\left|A_{\varepsilon}\right|_{\mathbb{C}}^{2}\right)
\end{aligned}
$$

Let us now argue on the bad set $\Omega \backslash U_{\varepsilon}^{\delta}$ by defining

$$
\begin{aligned}
& G_{1, \varepsilon}:=\left(I+\varepsilon \nabla \widehat{u}_{\varepsilon}\right)\left(I+\varepsilon \nabla u_{\varepsilon}\right)^{-1}, \\
& G_{2, \varepsilon}:=\left(I+\varepsilon z_{\varepsilon}\right)\left(I+\varepsilon \widehat{z}_{\varepsilon}\right)^{-1} .
\end{aligned}
$$

The energy bound (3.10), together with assumption (2.1a), implies that $I+\varepsilon \nabla u_{\varepsilon}$ is invertible almost everywhere. Note that $G_{1, \varepsilon}$ and $G_{2, \varepsilon}$ are chosen in such a way that $\widehat{F}_{\mathrm{el}, \varepsilon}=G_{1, \varepsilon} F_{\mathrm{el}, \varepsilon} G_{2, \varepsilon}$. We readily compute that

$$
G_{1, \varepsilon}-I=\nabla \psi_{\varepsilon}\left(\varphi_{\varepsilon}\right) \nabla \varphi_{\varepsilon}\left(I+\varepsilon \nabla u_{\varepsilon}\right)^{-1}-I=\nabla \psi_{\varepsilon}\left(\varphi_{\varepsilon}\right)-I=\varepsilon \nabla \tilde{u}\left(\varphi_{\varepsilon}\right)
$$

so that $\left\|G_{1, \varepsilon}-I\right\|_{L^{\infty}\left(\Omega \backslash U_{\varepsilon}^{\delta} ; \mathbb{R}^{d \times d}\right)}=\varepsilon\left\|\nabla \tilde{u}\left(\varphi_{\varepsilon}\right)\right\|_{L^{\infty}\left(\Omega \backslash U_{\varepsilon}^{\delta} ; \mathbb{R}^{d \times d}\right)} \leq c \varepsilon$. Moreover,

$$
G_{2, \varepsilon}= \begin{cases}\exp (-\varepsilon \tilde{z}) & \text { on }\left(\Omega \backslash U_{\varepsilon}^{\delta}\right) \cap \Omega_{\varepsilon}, \\ I & \text { on } \Omega \backslash\left(U_{\varepsilon}^{\delta} \cup \Omega_{\varepsilon}\right) .\end{cases}
$$

Hence, $\left\|G_{2, \varepsilon}-I\right\|_{\mathrm{L}^{\infty}\left(\Omega \backslash U_{\varepsilon}^{\delta} ; \mathbb{R}_{\mathrm{dev}}^{d \times d}\right)} \leq c \varepsilon$ as well.

Next, estimate (4.1) and bound (3.10) allow us to control the elastic part of the energy on the bad set $\Omega \backslash U_{\varepsilon}^{\delta}$ (where $\nabla u_{\varepsilon}$ and $z_{\varepsilon}$ are not under control) by cancellation. For this 
we employ the multiplicative estimate (2.1d) provided in (4.1):

$$
\begin{aligned}
\int_{\Omega \backslash U_{\varepsilon}^{\delta}}\left(W_{\mathrm{el}}^{\varepsilon}\right. & \left.\left(\widehat{A}_{\varepsilon}\right)-W_{\mathrm{el}}^{\varepsilon}\left(A_{\varepsilon}\right)\right) d x=\frac{1}{\varepsilon^{2}} \int_{\Omega \backslash U_{\varepsilon}^{\delta}}\left(W_{\mathrm{el}}\left(\widehat{F}_{\mathrm{el}, \varepsilon}\right)-W_{\mathrm{el}}\left(F_{\mathrm{el}, \varepsilon}\right)\right) d x \\
& =\frac{1}{\varepsilon^{2}} \int_{\Omega \backslash U_{\varepsilon}^{\delta}}\left(W_{\mathrm{el}}\left(G_{1, \varepsilon} F_{\mathrm{el}, \varepsilon} G_{2, \varepsilon}\right)-W_{\mathrm{el}}\left(F_{\mathrm{el}, \varepsilon}\right)\right) d x \\
& \stackrel{(4.1)}{\leq} \frac{c_{7}}{\varepsilon^{2}} \int_{\Omega \backslash U_{\varepsilon}^{\delta}}\left(W_{\mathrm{el}}\left(F_{\mathrm{el}, \varepsilon}\right)+c_{8}\right)\left(\left|G_{1, \varepsilon}-I\right|+\left|G_{2, \varepsilon}-I\right|\right) d x \\
& \leq c_{7}\left(\frac{1}{\varepsilon^{2}} \int_{\Omega} W_{\mathrm{el}}\left(F_{\mathrm{el}, \varepsilon}\right) d x+\frac{c_{8}}{\varepsilon^{2}}\left|\Omega \backslash U_{\varepsilon}^{\delta}\right|\right)\left(\left\|G_{1, \varepsilon}-I\right\|_{\mathrm{L}^{\infty}}+\left\|G_{2, \varepsilon}-I\right\|_{\mathrm{L}^{\infty}}\right) \\
& \stackrel{(3.10) \&(3.16)}{\leq}\left(1+\frac{1}{c_{\mathrm{el}}^{2}(\delta)}\right) \varepsilon .
\end{aligned}
$$

Thus, we can control the difference of the energy contributions in the bad set $\Omega \backslash U_{\varepsilon}^{\delta}$ where the gradients are large.

Finally, by using convergences (3.21)-(3.22), inequality (3.23) on the good set $U_{\varepsilon}^{\delta}$, (3.24) on the bad set $\Omega \backslash U_{\varepsilon}^{\delta}$, and the $\mathrm{L}^{2}$ boundedness of $\widehat{A}_{\varepsilon}$ and $A_{\varepsilon}$, we conclude that

$$
\begin{aligned}
& \limsup _{\varepsilon \rightarrow 0}\left(\int_{\Omega} W_{\mathrm{el}}^{\varepsilon}\left(\widehat{A}_{\varepsilon}\right) d x-\int_{\Omega} W_{\mathrm{el}}^{\varepsilon}\left(A_{\varepsilon}\right) d x\right) \\
& \stackrel{(3.23)}{\leq} \limsup _{\varepsilon \rightarrow 0}\left(\frac{1}{2} \int_{U_{\varepsilon}^{\delta}}\left(\widehat{A}_{\varepsilon}-A_{\varepsilon}\right): \mathbb{C}\left(\widehat{A}_{\varepsilon}+A_{\varepsilon}\right) d x+c \delta\right. \\
& \left.+\int_{\Omega \backslash U_{\varepsilon}^{\delta}}\left(W_{\mathrm{el}}^{\varepsilon}\left(\widehat{A}_{\varepsilon}\right)-W_{\mathrm{el}}^{\varepsilon}\left(A_{\varepsilon}\right)\right) d x\right) \\
& \stackrel{(3.24)}{\leq} \limsup _{\varepsilon \rightarrow 0}\left(\frac{1}{2} \int_{U_{\varepsilon}^{\delta}}\left(\widehat{A}_{\varepsilon}-A_{\varepsilon}\right): \mathbb{C}\left(\widehat{A}_{\varepsilon}+A_{\varepsilon}\right) d x+c \delta+c\left(1+\frac{1}{c_{\mathrm{el}}^{2}(\delta)}\right) \varepsilon\right) \\
& \leq \frac{1}{2} \int_{\Omega}(\nabla \tilde{u}-\tilde{z}): \mathbb{C}\left(\nabla\left(\widehat{u}_{0}+u_{0}\right)-\left(\widehat{z}_{0}+z_{0}\right)\right) d x+c \delta \\
& =\int_{\Omega}\left|\nabla \widehat{u}_{0}^{\mathrm{sym}}-\widehat{z}_{0}^{\mathrm{sym}}\right|_{\mathbb{C}}^{2} d x-\int_{\Omega}\left|\nabla u_{0}^{\mathrm{sym}}-z_{0}^{\mathrm{sym}}\right|_{\mathbb{C}}^{2} d x+c \delta
\end{aligned}
$$

where we have made use of relation (2.3).

Step 4: Upper bound on the hardening energy term. Let us now turn our attention to the hardening part of the energy. On the good set $Z_{\varepsilon}^{\delta}$ we have

$$
\begin{aligned}
W_{\mathrm{h}}^{\varepsilon}\left(\widehat{z}_{\varepsilon}\right)-W_{\mathrm{h}}^{\varepsilon}\left(z_{\varepsilon}\right) & \leq\left|\widehat{z}_{\varepsilon}\right|_{\mathbb{H}}^{2}-\left|z_{\varepsilon}\right|_{\mathbb{H}}^{2}+\delta\left(\left|\widehat{z}_{\varepsilon}\right|_{\mathbb{H}}^{2}+\left|z_{\varepsilon}\right|_{\mathbb{H}}^{2}\right) \\
& =\frac{1}{2}\left(\widehat{z}_{\varepsilon}-z_{\varepsilon}\right): \mathbb{H}\left(\widehat{z}_{\varepsilon}+z_{\varepsilon}\right)+\delta\left(\left|\widehat{z}_{\varepsilon}\right|_{\mathbb{H}}^{2}+\left|z_{\varepsilon}\right|_{\mathbb{H}}^{2}\right) .
\end{aligned}
$$

As regards the bad set $\Omega \backslash Z_{\varepsilon}^{\delta}$ one has

$$
W_{\mathrm{h}}^{\varepsilon}\left(\widehat{z}_{\varepsilon}\right)-W_{\mathrm{h}}^{\varepsilon}\left(z_{\varepsilon}\right)= \begin{cases}\frac{1}{\varepsilon^{2}} \widetilde{W}_{\mathrm{h}}\left(\exp (\varepsilon \tilde{z})\left(I+\varepsilon z_{\varepsilon}\right)\right)-\frac{1}{\varepsilon^{2}} \widetilde{W}_{\mathrm{h}}\left(I+\varepsilon z_{\varepsilon}\right) & \text { on }\left(\Omega \backslash Z_{\varepsilon}^{\delta}\right) \cap \Omega_{\varepsilon}, \\ 0 & \text { on } \Omega \backslash\left(Z_{\varepsilon}^{\delta} \cup \Omega_{\varepsilon}\right) .\end{cases}
$$


Hence, by exploiting the local Lipschitz continuity of $\widetilde{W}_{\mathrm{h}}$ we have

$$
\begin{aligned}
\int_{\Omega \backslash Z_{\varepsilon}^{\delta}}\left(W_{\mathrm{h}}^{\varepsilon}\left(\widehat{z}_{\varepsilon}\right)-W_{\mathrm{h}}^{\varepsilon}\left(z_{\varepsilon}\right)\right) d x & \leq \frac{c}{\varepsilon^{2}} \int_{\Omega \backslash Z_{\varepsilon}^{\delta}}|\exp (\varepsilon \tilde{z})-I|\left|I+\varepsilon z_{\varepsilon}\right| d x \\
& \leq \frac{c}{\varepsilon^{2}}\left|\Omega \backslash Z_{\varepsilon}^{\delta}\right| \frac{c \varepsilon}{c_{\mathrm{h}}^{2}(\delta)} \stackrel{(3.17)}{\leq} \frac{c \varepsilon}{c_{\mathrm{h}}^{2}(\delta)} .
\end{aligned}
$$

Finally, owing to convergences (3.12)-(3.13) we compute that

$$
\begin{aligned}
\limsup _{\varepsilon \rightarrow 0}\left(\int_{\Omega} W_{\mathrm{h}}^{\varepsilon}\left(\widehat{z}_{\varepsilon}\right) d x-\int_{\Omega} W_{\mathrm{h}}^{\varepsilon}\left(z_{\varepsilon}\right) d x\right) \\
\stackrel{(3.26)}{\leq} \limsup _{\varepsilon \rightarrow 0}\left(\int_{Z_{\varepsilon}^{\delta}} \frac{1}{2}\left(\widehat{z}_{\varepsilon}-z_{\varepsilon}\right): \mathbb{H}\left(\widehat{z}_{\varepsilon}+z_{\varepsilon}\right) d x+c \delta\right. \\
\left.\quad+\int_{\Omega \backslash Z_{\varepsilon}^{\delta}}\left(W_{\mathrm{h}}^{\varepsilon}\left(\widehat{z}_{\varepsilon}\right)-W_{\mathrm{h}}^{\varepsilon}\left(z_{\varepsilon}\right)\right) d x\right) \\
\stackrel{(3.27)}{\leq} \limsup _{\varepsilon \rightarrow 0}\left(\int_{Z_{\varepsilon}^{\delta}} \frac{1}{2}\left(\widehat{z}_{\varepsilon}-z_{\varepsilon}\right): \mathbb{H}\left(\widehat{z}_{\varepsilon}+z_{\varepsilon}\right) d x+c \delta+\frac{c \varepsilon}{c_{\mathrm{el}}^{2}(\delta)}\right) \\
\quad=\int_{\Omega} \frac{1}{2} \tilde{z}: \mathbb{H}\left(\widehat{z}_{0}+z_{0}\right) d x+c \delta=\int_{\Omega}\left|\widehat{z}_{0}\right|_{\mathbb{H}}^{2} d x-\int_{\Omega}\left|z_{0}\right|_{\mathbb{H}}^{2} d x+c \delta .
\end{aligned}
$$

Step 5: Conclusion of the proof. By collecting (3.25) and (3.28), and recalling that $\left\langle\ell(t), u_{\varepsilon}-\widehat{u}_{\varepsilon}\right\rangle \rightarrow\left\langle\ell(t), u_{0}-\widehat{u}_{0}\right\rangle$ we deduce that

$$
\limsup _{\varepsilon \rightarrow 0}\left(\mathcal{E}_{\varepsilon}\left(t, \widehat{u}_{\varepsilon}, \widehat{z}_{\varepsilon}\right)-\mathcal{E}_{\varepsilon}\left(t, u_{\varepsilon}, z_{\varepsilon}\right)\right) \leq \mathcal{E}_{0}\left(t, \widehat{u}_{0}, \widehat{z}_{0}\right)-\mathcal{E}_{0}\left(t, u_{0}, z_{0}\right)+c \delta .
$$

Finally, the assertion (3.11) follows by taking $\delta \rightarrow 0$ and employing (3.20).

\subsection{Proof of Theorem 2.2}

Owing to the above $\Gamma$-liminf and mutual-recovery-sequence results, the proof of Theorem 2.2 now follows along the lines of the general theory of [MRS08]. We just sketch the main points of the argument and refer the reader to [MRS08] for the details.

Let $\left(u_{\varepsilon}, z_{\varepsilon}\right)$ be a sequence of finite-plasticity solutions. The coercivity of the energy (3.1) entails an a priori bound on $\left(u_{\varepsilon}, z_{\varepsilon}\right)$. In particular, we have the following.

Corollary 3.7 (A priori bound). There exists $c>0$ such that all finite-plasticity solutions $\left(u_{\varepsilon}, z_{\varepsilon}\right)$ satisfy

$\forall t \in[0, T]: \quad\left\|u_{\varepsilon}(t)\right\|_{\mathrm{H}^{1}}+\left\|z_{\varepsilon}(t)\right\|_{\mathrm{L}^{2}}+\left\|\varepsilon z_{\varepsilon}(t)\right\|_{\mathrm{L}^{\infty}}+\operatorname{Diss}_{\mathcal{D}_{\varepsilon}}\left(z_{\varepsilon} ;[0, t]\right) \leq c$.

Proof. We exploit the energy balance (2.16) and the bound (3.1) to deduce that, for all $t \in[0, T]$ 


$$
\begin{aligned}
\| \nabla u_{\varepsilon}(t) & \left\|_{\mathrm{L}^{2}}^{2}+\right\| z_{\varepsilon}(t)\left\|_{\mathrm{L}^{2}}^{2}+\right\| \varepsilon z_{\varepsilon}(t) \|_{\mathrm{L}^{\infty}}^{2}+\operatorname{Diss}_{\mathcal{D}_{\varepsilon}}\left(z_{\varepsilon} ;[0, t]\right) \\
& \stackrel{(3.1)}{\leq} c\left(1+\mathcal{W}_{\varepsilon}\left(u_{\varepsilon}(t), z_{\varepsilon}(t)\right)\right)+\operatorname{Diss}_{\mathcal{D}_{\varepsilon}}\left(z_{\varepsilon} ;[0, t]\right) \\
& \leq c\left(1+\mathcal{E}_{\varepsilon}\left(t, u_{\varepsilon}(t), z_{\varepsilon}(t)\right)+\left\langle\ell(t), u_{\varepsilon}(t)\right\rangle+\operatorname{Diss}_{\mathcal{D}_{\varepsilon}}\left(z_{\varepsilon} ;[0, t]\right)\right) \\
& \stackrel{(2.16)}{=} c\left(1+\mathcal{E}_{\varepsilon}\left(0, u_{\varepsilon}^{0}, z_{\varepsilon}^{0}\right)+\left\langle\ell(t), u_{\varepsilon}(t)\right\rangle-\int_{0}^{t}\left\langle\dot{\ell}, u_{\varepsilon}\right\rangle d s\right) \\
& \leq c\left(1+\mathcal{E}_{\varepsilon}\left(0, u_{\varepsilon}^{0}, z_{\varepsilon}^{0}\right)+\|\ell(t)\|_{\mathrm{H}^{-1}}\left\|u_{\varepsilon}(t)\right\|_{\mathrm{H}^{1}}+\int_{0}^{t}\|\dot{\ell}\|_{\mathrm{H}^{-1}}\left\|u_{\varepsilon}\right\|_{\mathrm{H}^{1}} d s\right)
\end{aligned}
$$

so that the assertion follows by the Gronwall Lemma.

Owing to the a priori bound (3.29), we may now exploit the generalized version of Helly's Selection Principle in [MRS08, Thm. A.1] (consider also the comments thereafter) and deduce that, at least for some nonrelabeled subsequence, and all $s, t \in[0, T]$ with $s<t$,

$$
\begin{aligned}
& \delta_{0}(t):=\lim _{\varepsilon \rightarrow 0} \operatorname{Diss}_{\mathcal{D}_{\varepsilon}}\left(z_{\varepsilon} ;[0, t]\right), \\
& z_{\varepsilon}(t) \rightarrow z_{0}(t) \quad \text { weakly in } \mathcal{Z}, \\
& \operatorname{Diss}_{\mathcal{D}_{0}}\left(z_{0} ;[s, t]\right) \leq \delta_{0}(t)-\delta_{0}(s) .
\end{aligned}
$$

Moreover, letting $t \in[0, T]$ be fixed we may extract a further subsequence (still not relabeled, possibly depending on $t$ ) such that $u_{\varepsilon}(t) \rightarrow u_{*}$ weakly in $\mathcal{U}$. We now check that indeed $\left(u_{*}, z_{0}(t)\right) \in \mathcal{S}_{0}(t)$. To this end, by density it suffices to consider competitors $\left(\widehat{u}_{0}, \widehat{z}_{0}\right)=\left(u_{*}, z_{0}(t)\right)+(\tilde{u}, \tilde{z})$ with $(\tilde{u}, \tilde{z})$ smooth and compactly supported. By applying Lemma 3.6 we find a mutual recovery sequence $\left(\widehat{u}_{\varepsilon}, \widehat{z}_{\varepsilon}\right)$ such that

$$
\begin{aligned}
\mathcal{E}_{0}\left(t, \widehat{u}_{0}, \widehat{z}_{0}\right)-\mathcal{E}_{0}\left(t, u_{*}, z_{0}(t)\right)+\mathcal{D}_{0}\left(z_{0}(t), \widehat{z}_{0}\right) \\
\quad \geq \limsup _{\varepsilon \rightarrow 0}\left(\mathcal{E}_{\varepsilon}\left(t, \widehat{u}_{\varepsilon}, \widehat{z}_{\varepsilon}\right)-\mathcal{E}_{\varepsilon}\left(t, u_{\varepsilon}(t), z_{\varepsilon}(t)\right)+\mathcal{D}_{\varepsilon}\left(z_{\varepsilon}(t), \widehat{z}_{\varepsilon}\right)\right) \geq 0
\end{aligned}
$$

where the last inequality follows from the stability $(2.15)$ of $\left(u_{\varepsilon}(t), z_{\varepsilon}(t)\right)$. Hence, we have proved that $\left(u_{*}, z_{0}(t)\right) \in \mathcal{S}_{0}(t)$. Note that, given $z_{0}(t) \in \mathcal{Z}$, as the functional $u \in$ $\mathcal{U} \mapsto \mathcal{E}_{0}\left(t, u, z_{0}(t)\right)$ is uniformly convex, there exists a unique $u_{0}(t) \in \mathcal{U}$ such that $\left(u_{0}(t), z_{0}(t)\right) \in \mathcal{S}_{0}(t)$. From the fact that $\left(u_{*}, z_{0}(t)\right) \in \mathcal{S}_{0}(t)$ we conclude that $u_{*} \equiv$ $u_{0}(t)$. In particular $u_{\varepsilon}(t) \rightarrow u_{0}(t)$ weakly in $\mathcal{U}$ for all $t \in[0, T]$ and the whole sequence converges.

Let now a partition $0=t_{0}<t_{1}<\cdots<t_{N}=t$ be given. By passing to the lim inf in the energy balance (2.16) and using Lemmas 3.3 and 3.5 we get

$$
\begin{aligned}
\mathcal{E}_{0}\left(t, u_{0}(t), z_{0}(t)\right)+\sum_{i=1}^{N} \mathcal{D}_{0}\left(z_{0}\left(t_{i}\right), z_{0}\left(t_{i-1}\right)\right) \\
\quad \leq \liminf _{\varepsilon \rightarrow 0}\left(\mathcal{E}_{\varepsilon}\left(t, u_{\varepsilon}(t), z_{\varepsilon}(t)\right)+\sum_{i=1}^{N} \mathcal{D}_{\varepsilon}\left(z_{\varepsilon}\left(t_{i}\right), z_{\varepsilon}\left(t_{i-1}\right)\right)\right) \\
\quad \leq \liminf _{\varepsilon \rightarrow 0}\left(\mathcal{E}_{\varepsilon}\left(0, u_{\varepsilon}^{0}, z_{\varepsilon}^{0}\right)-\int_{0}^{t}\left\langle\dot{\ell}, u_{\varepsilon}\right\rangle d s\right)=\mathcal{E}_{0}\left(0, u_{0}^{0}, z_{0}^{0}\right)-\int_{0}^{t}\left\langle\dot{\ell}, u_{0}\right\rangle d s
\end{aligned}
$$


where for the last equality we have used (2.14) and the convergence of $u_{\varepsilon}$. Hence, the upper energy estimate follows by taking the sup over all partitions of the interval $[0, t]$. The lower energy estimate can classically be recovered from stability as in [Mie05, Prop. 2.7]. This proves that $\left(u_{0}, z_{0}\right)$ is a linearized-plasticity solution. In particular, as linearizedplasticity solutions are unique, the whole sequence $\left(u_{\varepsilon}, z_{\varepsilon}\right)$ converges and no extraction of subsequences is actually needed.

Along the lines of the proof of Theorem 2.2 (see also [MRS08, Thm. 3.1]) we also obtain the following convergences.

Corollary 3.8 (Improved convergences). Under the assumptions of Theorem 2.2 we have, for all $t \in[0, T]$,

$$
\begin{aligned}
\int_{\Omega}\left(W_{\mathrm{el}}^{\varepsilon}\left(A_{\varepsilon}\right)+W_{\mathrm{h}}^{\varepsilon}\left(z_{\varepsilon}\right)\right) d x & \rightarrow \int_{\Omega}\left(\left|\nabla u_{0}-z_{0}\right|_{\mathbb{C}}^{2}+\left|z_{0}\right|_{\mathbb{H}}^{2}\right) d x \\
\operatorname{Diss}_{\mathcal{D}_{\varepsilon}}\left(z_{\varepsilon} ;[0, t]\right) & \rightarrow \int_{0}^{t} R(\dot{z}) d s .
\end{aligned}
$$

In particular, owing to the energy convergence (3.31) we can deducing some strong convergence of finite-plasticity solutions to linearized-plasticity solutions.

Corollary 3.9 (Strong convergence). Under the assumptions of Theorem 2.2 for all $t \in$ $[0, T]$ we have $\left(u_{\varepsilon}(t), z_{\varepsilon}(t)\right) \rightarrow\left(u_{0}(t), z_{0}(t)\right)$ strongly in $\mathrm{W}^{1, p}\left(\Omega ; \mathbb{R}^{d}\right) \times \mathrm{L}^{p}\left(\Omega ; \mathbb{R}^{d \times d}\right)$ for all $p \in[1,2)$.

Proof. Let $v$ denote the Young measure generated by the sequence $\left(A_{\varepsilon}, z_{\varepsilon}\right)$ and define the measure $v^{\mathrm{sym}}\left(A^{s}, Z\right):=v\left(A^{s} \oplus \mathbb{R}_{\text {anti }}^{d \times d}, Z\right)$ for all Borel sets $\left(A^{s}, Z\right) \subset \mathbb{R}_{\mathrm{sym}}^{d \times d} \times \mathbb{R}^{d \times d}$. Note that $v^{\text {sym }}$ is indeed the Young measure generated by $\left(A_{\varepsilon}^{\text {sym }}, z_{\varepsilon}\right)$. By using the lowersemicontinuity Lemma 4.2 and the energy convergence (3.31) we deduce that

$$
\begin{aligned}
& \int_{\Omega}\left(\int_{\mathbb{R}_{\mathrm{sym}}^{d \times d} \times \mathbb{R}^{d \times d}}\left(\left|A^{\mathrm{sym}}\right|_{\mathbb{C}}^{2}+|z|_{\mathbb{H}}^{2}\right) d \nu_{x}^{\mathrm{sym}}\left(A^{\mathrm{sym}}, z\right)\right) d x \\
& \quad=\int_{\Omega}\left(\int_{\mathbb{R}^{d \times d} \times \mathbb{R}^{d \times d}}\left(|A|_{\mathbb{C}}^{2}+|z|_{\mathbb{H}}^{2}\right) d v_{x}(A, z)\right) d x \\
& \quad \leq \liminf _{\varepsilon \rightarrow 0} \int_{\Omega}\left(W_{\mathrm{el}}^{\varepsilon}\left(A_{\varepsilon}\right)+W_{\mathrm{h}}^{\varepsilon}\left(z_{\varepsilon}\right)\right) d x \stackrel{(3.31)}{=} \int_{\Omega}\left(\left|\nabla u_{0}-z_{0}\right|_{\mathbb{C}}^{2}+\left|z_{0}\right|_{\mathbb{H}}^{2}\right) d x .
\end{aligned}
$$

Recall from (3.7) that

$$
A_{\varepsilon}^{\mathrm{sym}}=\nabla u_{\varepsilon}^{\mathrm{sym}}-z_{\varepsilon}^{\mathrm{sym}}-\varepsilon\left(\nabla u_{\varepsilon} z_{\varepsilon}-\nabla u_{\varepsilon} w_{\varepsilon}\right)^{\mathrm{sym}}
$$

where the remainder term $\varepsilon\left(\nabla u_{\varepsilon} z_{\varepsilon}-\nabla u_{\varepsilon} w_{\varepsilon}\right)^{\text {sym }}$ converges strongly to 0 in $\mathrm{L}^{p}$ for all $p \in[1,2)$. Hence, the barycenter of $v^{\text {sym }}$ is clearly $\left(\nabla u_{0}^{\text {sym }}-z_{0}^{\text {sym }}, z_{0}\right)$. 
We readily check that the measure $v^{\text {sym }}$ is concentrated in its barycenter. Indeed, if this were not the case, by uniform convexity we would have

$$
\begin{aligned}
\int_{\Omega}\left(\left|\nabla u_{0}^{\mathrm{sym}}-z_{0}^{\mathrm{sym}}\right|_{\mathbb{C}}^{2}\right. & \left.+\left|z_{0}\right|_{\mathbb{H}}^{2}\right) d x \\
& <\int_{\Omega}\left(\int_{\mathbb{R}_{\mathrm{sym}}^{d \times d} \times \mathbb{R}^{d \times d}}\left(\left|A^{\mathrm{sym}}\right|_{\mathbb{C}}^{2}+|z|_{\mathbb{H}}^{2}\right) d \nu_{x}^{\mathrm{sym}}\left(A^{\mathrm{sym}}, z\right)\right) d x,
\end{aligned}
$$

contradicting (3.33). Here we have used positive definiteness from (2.5) and (2.9). As $v^{\text {sym }}$ is concentrated, we exploit [AGS08, Thm. 5.4.4.iii, p. 127] and deduce that

$$
\int_{\Omega} f\left(x, A_{\varepsilon}^{\mathrm{sym}}(x), z_{\varepsilon}(x)\right) d x \rightarrow \int_{\Omega}\left(\int_{\mathbb{R}_{\mathrm{sym}}^{d \times d} \times \mathbb{R}^{d \times d}} f\left(x, A^{\mathrm{sym}}, z\right) d \nu_{x}^{\mathrm{sym}}\left(A^{\mathrm{sym}}, z\right)\right) d x
$$

along with the choice

$$
f\left(x, A^{\mathrm{sym}}, z\right):=\left|\left(\nabla u_{0}^{\mathrm{sym}}(x)-z_{0}^{\mathrm{sym}}(x), z_{0}^{\mathrm{sym}}(x)\right)-\left(A^{\mathrm{sym}}-z^{\mathrm{sym}}, z\right)\right|^{p} .
$$

Hence, $\left(A_{\varepsilon}^{\mathrm{sym}}, z_{\varepsilon}\right) \rightarrow\left(\nabla u_{0}^{\mathrm{sym}}-z_{0}^{\mathrm{sym}}, z_{0}\right)$ strongly in $\mathrm{L}^{p}\left(\Omega ; \mathbb{R}_{\mathrm{sym}}^{d \times d}\right) \times \mathrm{L}^{p}\left(\Omega ; \mathbb{R}^{d \times d}\right)$ for all $p \in[1,2)$. In particular,

$$
\nabla u_{\varepsilon}^{\mathrm{sym}}=A_{\varepsilon}^{\mathrm{sym}}+z_{\varepsilon}^{\mathrm{sym}}+\varepsilon\left(\nabla u_{\varepsilon} z_{\varepsilon}-\nabla u_{\varepsilon} w_{\varepsilon}\right)^{\mathrm{sym}} \rightarrow \nabla u_{0}^{\mathrm{sym}} \quad \text { strongly in } \mathrm{L}^{p}
$$

for all $p \in[1,2)$, and the assertion follows by Korn's inequality.

\subsection{Sketch of the proof of Theorem 2.3}

The argument for Theorem 2.2 can be adapted to prove Theorem 2.3 as well. The only notable difference is that one has to cope with the fact that the piecewise constant interpolants $\left(\bar{u}_{\varepsilon}, \bar{z}_{\varepsilon}\right)$ of the approximate incremental minimizers need not be stable but rather just approximately stable. More precisely, from (2.17) and the triangle inequality we have

$$
\forall(\widehat{u}, \widehat{z}) \in \mathcal{Q}: \quad \mathcal{E}_{\varepsilon}(t, \widehat{u}, \widehat{z})-\mathcal{E}_{\varepsilon}\left(t, \bar{u}_{\varepsilon}(t), \bar{z}_{\varepsilon}(t)\right)+\mathcal{D}_{\varepsilon}\left(\bar{z}_{\varepsilon}(t), \widehat{z}\right) \geq-\tau_{\varepsilon} \alpha_{\varepsilon} .
$$

By coordinating with the sequence $\left(\bar{u}_{\varepsilon}(t), \bar{z}_{\varepsilon}(t)\right)$ a mutual recovery sequence $\left(\widehat{u}_{\varepsilon}, \widehat{z}_{\varepsilon}\right)$ via Lemma 3.6 (with $\left(\bar{u}_{\varepsilon}(t), \bar{z}_{\varepsilon}(t)\right)$ instead of $\left.\left(u_{\varepsilon}(t), z_{\varepsilon}(t)\right)\right)$ the lower bound (3.30) still follows as $\tau_{\varepsilon} \alpha_{\varepsilon} \rightarrow 0$. Hence, the stability of the limit can be recovered. Finally, improved and strong convergences in the spirit of Corollaries 3.8 and 3.9 can be established as well.

\section{Appendix}

\subsection{Estimate on left and right multiplication}

In the proof of Theorems 2.2-2.3 we have made use of the following estimate combining left and right multiplication. 
Lemma 4.1. Assume (2.1a) and (2.1d). Then

$$
\begin{aligned}
\exists c_{7}, c_{8}, \gamma>0 \forall G_{1}, G_{2} \in B_{\gamma}(I) \forall F \in \mathrm{GL}_{+}(d): \\
\quad\left|W_{\mathrm{el}}\left(G_{1} F G_{2}\right)-W_{\mathrm{el}}(F)\right| \leq c_{7}\left(W(F)+c_{8}\right)\left(\left|G_{1}-I\right|+\left|G_{2}-I\right|\right) .
\end{aligned}
$$

Proof. Following [Bal02, Lemma 2.5], we find positive constants $c_{0}, \widehat{c}_{0}, \gamma$ such that, for all $G \in B_{\gamma}(I)$ and all $F \in \mathrm{GL}_{+}(d)$,

$$
\begin{aligned}
& W_{\mathrm{el}}(G F) \leq \widehat{c}_{0} W_{\mathrm{el}}(F)+c_{0}, \quad W_{\mathrm{el}}(F G) \leq \widehat{c}_{0} W_{\mathrm{el}}(F)+c_{0}, \\
& \left|\partial_{F} W(G F) F^{\top}\right| \leq \widehat{c}_{0} W_{\mathrm{el}}(F)+c_{0}, \\
& \left|F^{\top} \partial_{F} W(F G)\right| \leq \widehat{c}_{0} W_{\mathrm{el}}(F)+c_{0} .
\end{aligned}
$$

For $s \in[0,1]$, let now $H_{j}(s):=(1-s) I+s G_{j}$ for $j=1,2$, and note that $H_{j} \in B_{\gamma}(I)$. As the derivative $H_{j}^{\prime}=G_{j}-I$ is constant we can compute that

$$
\begin{aligned}
& W_{\mathrm{el}}\left(G_{1} F G_{2}\right)-W_{\mathrm{el}}(F)=\int_{0}^{1} \frac{d}{d s} W_{\mathrm{el}}\left(H_{1}(s) F H_{2}(s)\right) d s \\
& \quad=\int_{0}^{1} \partial_{F} W_{\mathrm{el}}\left(H_{1} F H_{2}\right):\left(H_{1}^{\prime} F H_{2}+H_{1} F H_{2}^{\prime}\right) d s \\
& =\int_{0}^{1} \partial_{F} W_{\mathrm{el}}\left(H_{1} F H_{2}\right)\left(F H_{2}\right)^{\top} d s: H_{1}^{\prime}+\int_{0}^{1}\left(H_{1} F\right)^{\top} \partial_{F} W_{\mathrm{el}}\left(H_{1} F H_{2}\right) d s: H_{2}^{\prime} .
\end{aligned}
$$

We control the above right-hand side as

$$
\begin{aligned}
& \left|\int_{0}^{1} \partial_{F} W_{\mathrm{el}}\left(H_{1} F H_{2}\right)\left(F H_{2}\right)^{\top} d s: H_{1}^{\prime}\right| \stackrel{(4.3)}{\leq}\left(\int_{0}^{1}\left(\widehat{c_{0}} W_{\mathrm{el}}\left(F H_{2}\right)+c_{0}\right) d s\right)\left|G_{1}-I\right| \\
& \stackrel{(4.2)}{\leq}\left(\widehat{c}_{0}^{2} W_{\mathrm{el}}(F)+c_{0} \widehat{c}_{0}+c_{0}\right)\left|G_{1}-I\right|, \\
& \left|\int_{0}^{1}\left(H_{1} F\right)^{\top} \partial_{F} W_{\mathrm{el}}\left(H_{1} F H_{2}\right) d s: H_{2}^{\prime}\right| \stackrel{(4.4)}{\leq}\left(\int_{0}^{1}\left(\widehat{c}_{0} W_{\mathrm{el}}\left(H_{1} F\right)+c_{0}\right) d s\right)\left|G_{2}-I\right| \\
& \stackrel{(4.2)}{\leq}\left(\widehat{c}_{0}^{2} W_{\mathrm{el}}(F)+c_{0} \widehat{c}_{0}+c_{0}\right)\left|G_{2}-I\right|,
\end{aligned}
$$

whence the assertion follows.

\subsection{Lower-semicontinuity tool}

In Section 3 the following lower-semicontinuity lemma is used.

Lemma 4.2 (Lower semicontinuity). Let $f_{0}, f_{\varepsilon}: \mathbb{R}^{n} \rightarrow[0, \infty]$ be lower semicontinuous,

$$
\forall v_{0} \in \mathbb{R}^{n}: \quad f_{0}\left(v_{0}\right) \leq \inf \left\{\liminf _{\varepsilon \rightarrow 0} f_{\varepsilon}\left(v_{\varepsilon}\right) \mid v_{\varepsilon} \rightarrow v_{0}\right\},
$$


and $w_{\varepsilon} \rightarrow w_{0}$ weakly in $\mathrm{L}^{1}\left(\Omega ; \mathbb{R}^{n}\right)$. Denoting by $v$ the Young measure generated by $w_{\varepsilon}$ we have

$$
\int_{\Omega}\left(\int_{\mathbb{R}^{n}} f_{0}(w) d v_{x}(w)\right) d x \leq \liminf _{\varepsilon \rightarrow 0} \int_{\Omega} f_{\varepsilon}\left(w_{\varepsilon}\right) d x .
$$

In particular, if $f_{0}$ is convex we have

$$
\int_{\Omega} f_{0}\left(w_{0}\right) d x \leq \liminf _{\varepsilon \rightarrow 0} \int_{\Omega} f_{\varepsilon}\left(w_{\varepsilon}\right) d x .
$$

This lemma is in the same spirit of the results by Balder [Bal84a, Thm. 1] and Ioffe [Iof77] and can be proved via augmenting the variables by including the parameter $\varepsilon$. The reader is referred to [Ste08, Thm. 4.3, Cor. 4.4] or [MRS09, Lemma 3.1] for a proof in the case $d=1$. In the case of local uniform convergence, a proof can be found in [Li96].

Acknowledgments. We are gratefully indebted to the referee for her/his careful reading of the manuscript.

A. Mielke was partially supported by DFG within the Research Unit FOR 797 (subproject P5, Mie 459/5-2). U. Stefanelli was partially supported by FP7-IDEAS-ERC-StG Grant \#200497 BioSMA, the CNR-AVČR grant SmartMath, and the Alexander von Humboldt Foundation.

\section{References}

[ADD12] Agostiniani, V., Dal Maso, G., DeSimone, A.: Linear elasticity obtained from finite elasticity by $\Gamma$-convergence under weak coerciveness conditions. Ann. Inst. H. Poincaré Anal. Non Linéaire 29, 715-735 (2012) Zbl 1250.74008 MR 2971028

[AD11] Agostiniani, V., DeSimone, A.: $\Gamma$-convergence of energies for nematic elastomers in the small strain limit. Contin. Mech. Thermodyn. 23, 257-274 (2011) MR 2795609

[AGS08] Ambrosio, L., Gigli, N., Savaré, G.: Gradient Flows in Metric Spaces and in the Space of Probability Measures. 2nd ed., Lectures in Math. ETH Zürich, Birkhäuser, Basel (2008) Zbl 1145.35001 MR 2401600

[Bal84a] Balder, E. J.: A general approach to lower semicontinuity and lower closure in optimal control theory. SIAM J. Control Optim. 22, 570-598 (1984) Zbl 0549.49005 MR 0747970

[Bal84b] Ball, J. M.: Minimizers and the Euler-Lagrange equations. In: Trends and Applications of Pure Mathematics to Mechanics (Palaiseau, 1983), Lecture Notes in Phys. 195, Springer, Berlin, 1-4 (1984) Zbl 0547.73013 MR 0755716

[Bal02] Ball, J. M.: Some open problems in elasticity. In: P. Newton et al. (eds.), Geometry, Mechanics, and Dynamics (in honor of the 60th birthday of J. E. Marsden), Springer, New York, NY, 3-59 (2002) Zbl 1054.74008 MR 1919825

[BRM09] Bouchitté, G., Roubíček, T., Mielke, A.: A complete-damage model problem at small strains. Z. Angew. Math. Phys. 60, 205-236 (2009) Zbl 1238.74005 MR 2486153

[BSV07] Braides, A., Solci, M., Vitali, E.: A derivation of linear elastic energies from pair-interaction atomistic systems. Netw. Heterog. Media 2, 551-567 (2007) Zbl 1183.74017 MR 2318845

[CHM02] Carstensen, C., Hackl, K., Mielke, A.: Non-convex potentials and microstructures in finite-strain plasticity. Roy. Soc. London Proc. Ser. A Math. Phys. Eng. Sci. 458, 299317 (2002) Zbl 1008.74016 MR 1889770 
[DNP02] Dal Maso, G., Negri, M., Percivale, D.: Linearized elasticity as $\Gamma$-limit of finite elasticity. Set-Valued Anal. 10, 165-183 (2002) Zbl 1009.74008 MR 1926379

[FM06] Francfort, G., Mielke, A.: Existence results for a class of rate-independent material models with nonconvex elastic energies. J. Reine Angew. Math. 595, 55-91 (2006) Zbl 1101.74015 MR 2244798

[FPZ10] Freddi, L., Paroni, R., Zanini, C.: Dimension reduction of a crack evolution problem in a linearly elastic plate. Asymptot. Anal. 70, 101-123 (2010) Zbl 1219.35306 MR 2731652

[FJM02] Friesecke, G., James, R. D., Müller, S.: A theorem on geometric rigidity and the derivation of nonlinear plate theory from three-dimensional elasticity. Comm. Pure Appl. Math. 55, 1461-1506 (2002) Zbl 1021.74024 MR 1916989

[GP06a] Giacomini, A., Ponsiglione, M.: Discontinuous finite elements approximation of quasistatic crack growth in nonlinear elasticity. Math. Models Methods Appl. Sci. 16, $77-$ 118 (2006) Zbl 1092.35111 MR 2194982

[GP06b] Giacomini, A., Ponsiglione, M.: A $\Gamma$-convergence approach to stability of unilateral minimality properties in fracture mechanics and applications. Arch. Ration. Mech. Anal. 180, 399-447 (2006) Zbl 1089.74011 MR 2214962

[GN11] Gloria, A., Neukamm, S.: Commutability of homogeneization and linearization at identity in finite elasticity and applications. Ann. Inst. H. Poincaré Anal. Non Linéaire 28, 941-964 (2011) Zbl pre05992248 MR 2859933

[HR99] Han, W., Reddy, B. D.: Plasticity. Mathematical Theory and Numerical Analysis. Springer, New York (1999) Zbl 0926.74001 MR 1681061

[Hil50] Hill, R.: The Mathematical Theory of Plasticity. Clarendon Press, Oxford (1950) Zbl 0041.10802 MR 0037721

[Iof77] Ioffe, A. D.: On lower semicontinuity of integral functionals. I. SIAM J. Control Optim. 15, 521-538 (1977) Zbl 0361.46037 MR 0637234

[Joh76] Johnson, C.: Existence theorems for plasticity problems. J. Math. Pures Appl. 55, 431444 (1976) Zbl 0351.73049 MR 0438867

[KMR05] Kružík, M., Mielke, A., Roubíček, T.: Modelling of microstructure and its evolution in shape-memory-alloy single-crystals, in particular in CuAlNi. Meccanica 40, 389-418 (2005) Zbl 1106.74048 MR 2200210

[Lee69] Lee, E.: Elastic-plastic deformation at finite strains. J. Appl. Mech. 36, 1-6 (1969) Zbl 0179.55603

[Li96] Li, Z.: A theorem on lower semicontinuity of integral functionals. Proc. Roy. Soc. Edinburgh Sect. A 126, 363-374 (1996) Zbl 0849.49014 MR 1386868

[LM11] Liero, M., Mielke, A.: An evolutionary elastoplastic plate model derived via $\Gamma$ convergence. Math. Models Methods Appl. Sci. 21, 1961-1986(2011) Zbl 1232.35165 MR 2843026

[Lub90] Lubliner, J.: Plasticity Theory. Macmillan, New York (1990) Zbl 0745.73006

[MM09] Mainik, A., Mielke, A.: Global existence for rate-independent gradient plasticity at finite strain. J. Nonlinear Sci. 19, 221-248 (2009) Zbl 1173.49013 MR 2511255

[Mar75] Martins, J. B.: Plasticity. Fundamentals and General Results. MIT Press, Cambridge (1975)

[Mie03] Mielke, A.: Energetic formulation of multiplicative elasto-plasticity using dissipation distances. Contin. Mech. Thermodyn. 15, 351-382 (2003) Zbl 1068.74522 MR 1999280

[Mie04] Mielke, A.: Existence of minimizers in incremental elasto-plasticity with finite strains. SIAM J. Math. Anal. 36, 384-404 (2004) Zbl 1076.74012 MR 2111782 
[Mie05] Mielke, A.: Evolution of rate-independent systems. In: C. Dafermos and E. Feireisl (eds.), Handbook of Differential Equations, Evolutionary Equations, Volume 2, Elsevier, 461-559 (2005) Zbl 1120.47062 MR 2182832

[MM06] Mielke, A., Müller, S.: Lower semicontinuity and existence of minimizers in incremental finite-strain elastoplasticity. Z. Angew. Math. Mech. 86, 233-250 (2006) Zbl 1102.74006 MR 2205645

[MRS09] Mielke, A., Rossi, R., Savaré, G.: Modeling solutions with jumps for rate-independent systems on metric spaces. Discrete Contin. Dynam. Systems Ser. A 25, 585-615 (2009) Zbl 1170.49036 MR 2525194

[MR09] Mielke, A., Roubíček, T.: Numerical approaches to rate-independent processes and applications in inelasticity. Math. Model. Numer. Anal. 43, 399-428 (2009) Zbl 1166.74010 MR 2527399

[MRS08] Mielke, A., Roubíček, T., Stefanelli, U.: $\Gamma$-limits and relaxations for rate-independent evolutionary problems. Calc. Var. Partial Differential Equations 31, 387-416 (2008) Zbl pre05236593 MR 2366131

[MT04] Mielke, A., Theil, F.: On rate-independent hysteresis models. Nonlinear Differential Equations Appl. 11, 151-189 (2004) Zbl 1109.74040 MR 2211809

[MT05] Mielke, A., Timofte, A. M.: An energetic material model for time-dependent ferroelectric behavior: existence and uniqueness. Math. Models Appl. Sci. 29, 1393-1410 (2005) Zbl 1096.74017 MR 2247308

[MN11] Müller, S., Neukamm, S.: On the commutability of homogenization and linearization in finite elasticity. Arch. Ration. Mech. Anal. 201, 465-500 (2011) Zbl pre06101931 MR 2820355

[Neu10] Neukamm, S.: Homogeneization, linearization and dimensional reduction in elasticity with variational methods. PhD thesis, Technische Univ. München (2010)

[PT09] Paroni, R., Tomassetti, G.: A variational justification of linear elasticity with residual stress. J. Elast. 97, 189-206 (2009) Zbl 1188.74009 MR 2563158

[PT11] Paroni, R., Tomassetti, G.: From non-linear elasticity to linear elasticity with initial stress via $\Gamma$-convergence. Contin. Mech. Thermodyn. 23, 347-361 (2011) MR 2823756

[Rin08] Rindler, F.: Optimal control for nonconvex rate-independent evolution processes. SIAM J. Control Optim. 47, 2773-2794 (2008) Zbl 1176.49005 MR 2466091

[Rin09] Rindler, F.: Approximation of rate-independent optimal control problems. SIAM J. Numer. Anal. 47, 3884-3909 (2009) Zbl 1206.65173 MR 2576524

[RSZ09] Roubíček, T., Scardia, L., Zanini, C.: Quasistatic delamination problem. Contin. Mech. Thermodyn. 21, 223-235 (2009) Zbl 1179.74130 MR 2529453

[SZ11] Scardia, L., Zeppieri, C. I.: Gradient theory for plasticity as the $\Gamma$-limit of a nonlinear dislocation energy. http://cvgmt.sns.it/cgi/get.cgi/papers/scazep10/ (2011)

[Sch08] Schmidt, B.: Linear $\Gamma$-limits of multiwell energies in nonlinear elasticity theory. Contin. Mech. Thermodyn. 20, 375-396 (2008) Zbl 1160.74321 MR 2461715

[Sch09] Schmidt, B.: On the derivation of linear elasticity from atomistic models. Netw. Heterog. Media 4, 789-812 (2009) Zbl 1183.74020 MR 2552170

[Ste08] Stefanelli, U.: The Brezis-Ekeland principle for doubly nonlinear equations. SIAM J. Control Optim. 47, 1615-1642 (2008) Zbl 1194.35214 MR 2425653

[Suq81] Suquet, P.-M.: Sur les équations de la plasticité: existence et régularité des solutions. J. Mécanique 20, 3-39 (1981) Zbl 0474.73030 MR 0618942

[TM10] Thomas, M., Mielke, A.: Damage of nonlinearly elastic materials at small strainexistence and regularity results. Z. Angew. Math. Mech. 90, 88-112 (2010) Zbl 1191.35159 MR 2640367

[Tim09] Timofte, A.: Homogenization for a nonlinear ferroelectric model. Asymptot. Anal. 61, 177-194 (2009) Zbl 1173.35337 MR 2499115 